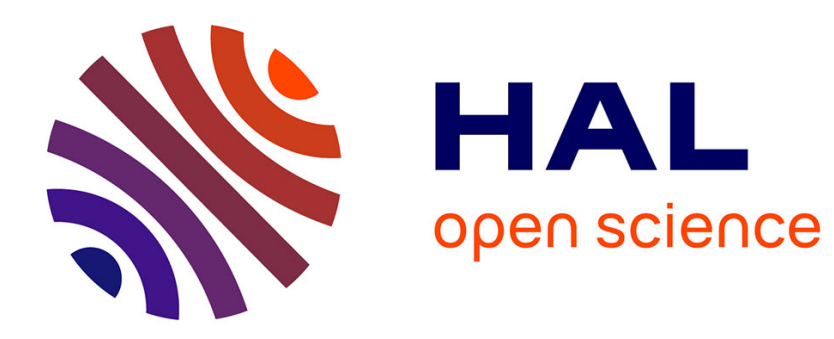

\title{
Oscillations localisées sur les diviseurs
}

Régis de La Bretèche, Gerald Tenenbaum

\section{To cite this version:}

Régis de La Bretèche, Gerald Tenenbaum. Oscillations localisées sur les diviseurs. Journal of the London Mathematical Society, 2012, 85, pp.669-693. hal-01281336

\section{HAL Id: hal-01281336 https://hal.science/hal-01281336}

Submitted on 2 Mar 2016

HAL is a multi-disciplinary open access archive for the deposit and dissemination of scientific research documents, whether they are published or not. The documents may come from teaching and research institutions in France or abroad, or from public or private research centers.
L'archive ouverte pluridisciplinaire HAL, est destinée au dépôt et à la diffusion de documents scientifiques de niveau recherche, publiés ou non, émanant des établissements d'enseignement et de recherche français ou étrangers, des laboratoires publics ou privés. 


\title{
Oscillations localisées sur les diviseurs*
}

\author{
R. de la Bretèche \& G. Tenenbaum
}

Abstract. Let $f$ be a real arithmetic function and

$$
\Delta(n, f):=\sup _{u \in \mathbb{R}, 0 \leqslant v \leqslant 1}\left|\sum_{\substack{d \mid n \\ \mathrm{e}^{u}<d \leqslant \mathrm{e}^{u+v}}} f(d)\right|
$$

denote the corresponding generalization of Hooley's Delta-function. We investigate weighted moments of $\Delta(n ; f)$ for oscillating functions $f$, typical cases being those of a non principal Dirichlet character or of the Möbius function. We obtain, in particular, sharp bounds up to factors $(\log x)^{o(1)}$ for all weighted finite integral, even moments computed on the integers not exceeding $x$. This is the key step to the proof, given in a subsequent work, of Manin's conjecture, in the strong form conjectured by Peyre and with an effective remainder term, for all Châtelet surfaces. The proof of the main results rest upon a genuinely new approach for Hooley-type functions.

Keywords: Hooley's Delta-function, divisors, Dirichlet characters, Möbius function, moments of arithmetic functions, concentration functions, Châtelet surfaces, Manin's conjecture, Peyre's constant, count of lattice points over algebraic varieties.

\section{Sommaire}

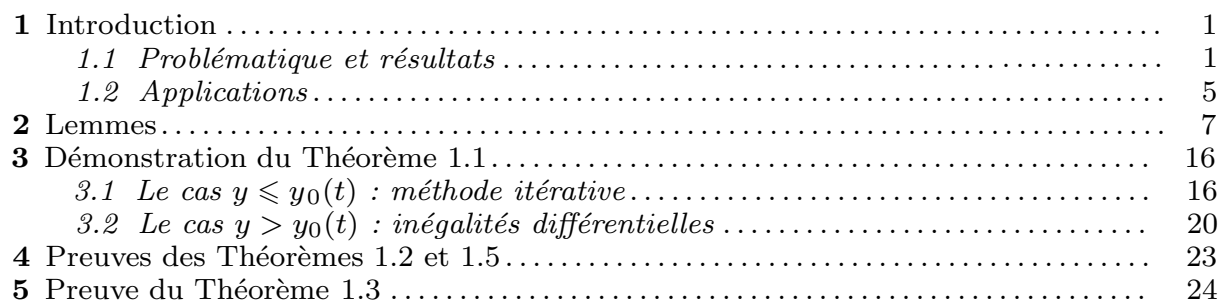

\section{Introduction}

\subsection{Problématique et résultats}

Étant donnée une fonction arithmétique $f$, posons

$$
\begin{aligned}
\Delta(n, f ; u, v) & :=\sum_{\substack{d \mid n \\
\mathrm{e}^{u}<d \leqslant \mathrm{e}^{u+v}}} f(d), \quad \Delta(n ; u, v):=\Delta(n, \mathbf{1} ; u, v) \quad\left(n \in \mathbb{N}^{*}, u \in \mathbb{R}, v>0\right), \\
\Delta(n, f) & :=\sup _{u \in \mathbb{R}, 0 \leqslant v \leqslant 1}|\Delta(n, f ; u, v)|, \quad \Delta(n):=\sup _{\substack{u \in \mathbb{R} \\
0 \leqslant v \leqslant 1}} \Delta(n, \mathbf{1} ; u, v)=\sup _{u \in \mathbb{R}} \Delta(n ; u, 1) .
\end{aligned}
$$

Ainsi, $n \mapsto \Delta(n)$ n'est autre que la fonction, aujourd'hui classique, introduite par Hooley [11] en 1979 — voir aussi [19]. À la base de sa «nouvelle technique», cette mesure de la concentration des diviseurs s'est révélée un outil arithmétique très performant et les estimations obtenues dans la littérature, tant en ordre normal qu'en ordre moyen, ont constitué les étapes décisives de nombreuses applications dans des branches variées de la théorie des nombres. Il est à noter, en particulier, que la conjecture d'Erdös (voir par exemple [6]) selon laquelle l'inégalité $\Delta(n)>1$ a lieu sur une suite de densité naturelle unité a été établie par Maier et Tenenbaum en 1984 [13] après avoir résisté pendant presque un demi-siècle. On pourra consulter l'article [14] pour une vue d'ensemble actualisée de cet aspect de la question.

* Nous incluons ici certaines corrections relativement à la version publiée.

2010 Mathematics Subject Classification: primary 11N37, 11L40, 11N56 ; secondary 11D45. 
De récentes études ont mis en évidence que les généralisations $\Delta(n, f)$ apparaissent naturellement dans des applications d'envergure lorsque $f$ est une fonction arithmétique oscillante, comme un caractère de Dirichlet réel non principal ou la fonction de Möbius. De plus, il s'avère alors souvent nécessaire de considérer des moyennes pondérées dont les coefficients sont eux-mêmes susceptibles de présenter des fluctuations arithmétiques, comme le nombre des solutions modulo $n$ d'une équation algébrique en plusieurs variables.

Nous nous proposons ici de prouver, dans un cadre relativement général, les estimations requises au développement de ces applications.

Notre résultat principal ${ }^{(1)}$ concerne l'évaluation asymptotique des moments pairs de $\Delta(n, f)$, pondérés par une fonction arithmétique $g$. Nous commençons par décrire les hypothèses effectuées sur le poids $g$.

Pour $A>0, c>0, \eta \in] 0,1\left[\right.$, nous introduisons la classe $\mathcal{M}_{A}(c, \eta)$ des fonctions arithmétiques $g$ multiplicatives, positives ou nulles, satisfaisant, pour une constante convenable $A>0$, aux majorations

$$
\begin{aligned}
g\left(p^{\nu}\right) & \leqslant A^{\nu} \quad(\nu \geqslant 1) \\
(\forall \varepsilon>0) \quad g(n) & \ll_{\varepsilon} n^{\varepsilon},
\end{aligned}
$$

et, pour une constante $y=y(g) \geqslant 0$ convenable, à la relation asymptotique

$$
\sum_{p \leqslant x} g(p)=y \operatorname{li}(x)+O\left(x \mathrm{e}^{-2 c(\log x)^{\eta}}\right) .
$$

Ici et dans la suite, nous réservons la lettre $p$ pour désigner un nombre premier. Nous notons $\mathcal{P}$ l'ensemble de tous les nombres premiers.

Pour tout caractère de Dirichlet réel non principal $\chi$ nous désignons par $\mathcal{M}_{A}(\chi ; c, \eta)$ la sous-classe de $\mathcal{M}_{A}(c, \eta)$ constituée des fonctions arithmétiques $g$ vérifiant, pour une constante $z=z(g) \in \mathbb{R}$ convenable, la relation asymptotique

$$
\sum_{p \leqslant x} \chi(p) g(p)=z \operatorname{li}(x)+O\left(x \mathrm{e}^{-c(\log x)^{\eta}}\right)
$$

Les relations (1.3) et (1.4) signifient essentiellement, sous une forme faible, que la suite $\{g(p)\}_{p \in \mathcal{P}}$ se comporte en moyenne comme une constante sur les classes de congruence modulo le conducteur de $\chi$. Ainsi, si $g$ vérifie $(1 \cdot 2)$ et $(1 \cdot 3)$, et s'il existe un entier $m$ tel que $g(p)$ soit effectivement constant sur chaque classe de congruence de module $m$, alors $g \in \mathcal{M}_{A}(\chi ; 1, \eta)$ pour tout caractère non principal $\chi$ et tout $\eta<\frac{3}{5}$, avec

$$
y:=\frac{1}{\varphi(m)} \sum_{\substack{1 \leqslant r \leqslant m \\(r, m)=1}} \gamma(r), \quad z:=\frac{1}{\varphi([m, b])} \sum_{\substack{1 \leqslant a \leqslant b \\(a, b)=1}} \chi(a) \sum_{\substack{1 \leqslant r \leqslant m \\(r, m)=1 \\ r \equiv a(\bmod (m, b))}} \gamma(r),
$$

où $\gamma(r)$ est la valeur commune des $g(p)$ lorsque $p \equiv r(\bmod m)$ et $b$ est le module de $\chi$. Ce résultat découle immédiatement de la validité d'une région sans zéro de type VinogradovKorobov pour les séries $L$ de Dirichlet — cf., par exemple, [15].

Nous nous proposons de montrer le résultat suivant. Nous posons

$$
y_{0}=y_{0}(t):=t /\left(2^{2 t-1}-1\right) \quad(t \geqslant 1)
$$

et utilisons librement la notation $x^{+}:=\max \{x, 0\}(x \in \mathbb{R})$.

1. Il s'agit d'une version légèrement plus précise de résultats communs exposés par le second auteur à

l'école de printemps Conference on Analytic Number Theory, Göttingen, avril 2010. 
Théorème 1.1. Soient $\chi$ un caractère de Dirichlet réel non principal, $A>0, c>0, t \geqslant 1$, $\eta \in] 0,1\left[\right.$ et $g \in \mathcal{M}_{A}(\chi ; c, \eta)$ telle que $y=y(g)>0, z(g) \leqslant 2^{1-2 t}\left\{1+t\left(1-y / y_{0}\right)^{+}\right\}$. Il existe une constante $\alpha=\alpha(g, \chi ; c, t, \eta)>0$ telle que l'on ait

$$
\begin{aligned}
\mathfrak{S}_{t}(x, \chi ; g) & :=\sum_{n \leqslant x} g(n) \Delta(n, \chi)^{2 t} \\
& \ll x \mathrm{e}^{\alpha \sqrt{\log _{2} x \log _{3} x}}(\log x)^{y-1+\left(2^{2 t-1} y-y-t\right)^{+}}
\end{aligned}
$$

Le facteur $\log _{3} x$ peut être omis dans le membre de droite lorsque $y>y_{0}(t)$.

Si $t$ est entier, $t \geqslant 2, y(g)>\max \left\{2^{1-t}, 2 y_{0}(t)\right\}, z(g) \leqslant 0$, nous avons en outre

$$
\mathfrak{S}_{t}(x, \chi ; g) \ll x \mathrm{e}^{\alpha \sqrt{\log _{2} x}}(\log x)^{2^{2 t-1} y-2 t-1} \quad(x \geqslant 16) .
$$

Remarques. (i) On a $2^{1-t}>2 y_{0}(t)$ si, et seulement si, $t \geqslant 3$. Lorsque $t=2$, la condition de validité de (1.6) est donc $y>\frac{4}{7}$. Les hypothèses $y>2^{1-t}$ et $z \leqslant 0$ impliquant (1.6) lorsque $t$ est entier pourraient être sensiblement affaiblies en raffinant le Lemme 2.6 infra. Nous n'avons pas recherché le meilleur résultat possible dans cette direction.

(ii) Nous avons concentré notre étude sur le cas $t \geqslant 1$, qui reflète le plus nettement le phénomène de compensation spécifique aux oscillations localisées sur les diviseurs. Cependant, lorsque $\frac{1}{2} \leqslant t \leqslant 1$, nos estimations fournissent immédiatement, via l'inégalité de Hölder, l'existence d'une constante $\alpha=\alpha(g, \chi ; c, t, \eta)>0$ telle que l'on ait, pour $y(g)>0$ et $z(g) \leqslant 1-\min (1, y) / 2$,

$$
\mathfrak{S}_{t}(x, \chi ; g) \ll x \mathrm{e}^{\alpha \sqrt{\log _{2} x \log _{3} x}}(\log x)^{y-1+t(y-1)^{+}} \quad(x \geqslant 16) .
$$

Le facteur $\log _{3} x$ peut être omis dans le membre de droite lorsque $y>1$.

(iii) La condition $z(g) \leqslant Z=Z(y, t):=2^{1-2 t}\left\{1+t\left(1-y / y_{0}\right)^{+}\right\}$est génériquement équivalente à

$$
\sum_{n \leqslant x} \chi(n) g(n) \ll x(\log x)^{Z-1} .
$$

$\mathrm{Si}$, par exemple, on choisit $g(p)=y+\varepsilon \chi(p)$ avec $0<\varepsilon \leqslant Z$, alors $z(g)=\varepsilon$ et, en vertu d'un théorème de type Selberg-Delange (voir [21], th. III.5.2) la somme (1.8) vaut $\{C+o(1)\} x(\log x)^{\varepsilon-1}$ où $C$ est une constante positive dépendant de $\varepsilon, y$ et $\chi$.

(iv) Le cas $t=1,0<y<1$, du Théorème 1.1 est particulièrement significatif. La condition $Z=1-y / 2$ reflète alors, sous une forme simple, la souplesse de nos hypothèses. Ainsi, posons par exemple $g\left(2^{\nu}\right)=0(\nu \geqslant 1)$, et, lorsque $p>2, g(p)=\frac{2}{3}\{1+\chi(p)\}, g\left(p^{\nu}\right)=0(\nu>1)$. En choisissant alors $\chi$ comme le caractère non principal de module 4 , nous obtenons

$$
\sum_{n \leqslant x} g(n) \Delta(n, \chi)^{2}=\sum_{\substack{n \leqslant x \\ n \in E}} \mu(n)^{2}\left(\frac{4}{3}\right)^{\omega(n)} \Delta(n)^{2}=x(\log x)^{-1 / 3+o(1)} \quad(x \rightarrow \infty),
$$

où $E$ désigne l'ensemble des entiers impairs dont tous les facteurs premiers sont congrus à 1 modulo 4 et $\omega(n)$ désigne le nombre des facteurs premiers distincts d'un entier naturel $n$. La minoration implicite dans (1.9) provient de l'inégalité (4.1) infra. On voit ainsi que l'inégalité $y(g) \leqslant \frac{2}{3}$ apparaissant, lorsque $t=1$, dans les hypothèses du Théorème 1.1 sous la contrainte supplémentaire $z(g)=y(g)$ est optimale.

Il est intéressant de comparer la majoration (1.5) à celle des moments de la fonction $\Delta$. Il est en effet établi dans [8] que nous avons

$$
\sum_{n \leqslant x} y^{\omega(n)} \Delta(n)^{t} \ll x(\log x)^{y-1+\left(2^{t} y-y-t\right)^{+}} \mathrm{e}^{\{2 \sqrt{t}+o(1)\} \sqrt{\log _{2} x \log _{3} x}}
$$


pour tous $y, t$ fixés tels que $t \geqslant 1, y>0 .^{(2)}$ De plus, l'exposant de $\log x$ dans cette majoration est optimal. Ainsi, pour le choix $y=1, t=1$, nous constatons que $\Delta(n, \chi)$ se comporte en moyenne comme $\sqrt{\Delta(n)}$ : cela signifie que, dans les intervalles $\left.] \mathrm{e}^{u}, \mathrm{e}^{u+1}\right]$ où le nombre des diviseurs de $n$ est proche du maximum, les compensations dues aux oscillations du caractère sont de nature statistique.

Nous conjecturons que l'exposant de $\log x$ dans (1.5) est optimal lorsque $t$ est proche de 1 et que la majoration (1.6) est valable et optimale - à un facteur $(\log x)^{o(1)}$ près — pour $t \geqslant t_{0}, y(g) \geqslant y_{1}(t), z(g) \leqslant 0$, où $t_{0} \leqslant 2$ et $y_{1}(t)$ est une fonction continue de $t$ vérifiant $y_{1}(t) \geqslant 2 y_{0}(t)$ avec égalité pour $t$ assez grand. Ces hypothèses sont certainement confirmées lorsque $t \in \mathbb{N}^{*}$, comme l'atteste l'énoncé suivant.

Théorème 1.2. Soient $\chi$ un caractère de Dirichlet réel non principal, $y>0$, et $t \geqslant 1$. Nous avons

$$
\sum_{n \leqslant x} \mu(n)^{2} y^{\omega(n)} \Delta(n ; \chi)^{2 t} \gg x(\log x)^{y-1+\max \left(2^{t} y-y-t, 2^{2 t-1} y-y-2 t, 0\right)} .
$$

Ainsi, nous pouvons écrire

$$
\sum_{n \leqslant x} \mu(n)^{2} y^{\omega(n)} \Delta(n, \chi)^{2}=x(\log x)^{y-1+(y-1)^{+}+o(1)} \quad(y>0),
$$

et, lorsque $t \in \mathbb{N}, t \geqslant 2, y>\max \left\{2^{1-t}, 2 t /\left(2^{2 t-1}-1\right)\right\}$,

$$
\sum_{n \leqslant x} \mu(n)^{2} y^{\omega(n)} \Delta(n, \chi)^{2 t}=x(\log x)^{2^{2 t-1} y-2 t-1+o(1)} .
$$

De plus, la relation (1.10) apporte l'information que l'exposant 2 est maximal sous la contrainte que le moment correspondant de $\Delta(n, \chi)$ soit $(\log x)^{o(1)}$ lorsque $x \rightarrow \infty$.

Nous observons que la même méthode permettrait, au prix de quelques complications techniques standard que nous avons préféré éviter ici, de montrer que la minoration (1.10) persiste, dans les hypothèses du Théorème 1.1, en remplaçant $y^{\omega(n)}$ par $g(n)$ dans le membre de gauche et $y$ par $y(g)$ dans celui de droite. Cette remarque est également valable pour le Théorème 1.5 infra.

Nos méthodes sont transposables sans difficulté au cas où le caractère non principal $\chi$ est remplacé par une fonction multiplicative oscillante suffisamment régulière sur l'ensemble des nombres premiers. À titre d'illustration, nous énonçons le résultat obtenu dans le cas de la fonction de Möbius.

Nous posons

$$
\lambda(t):=\frac{1}{2 \pi} \int_{-\pi}^{\pi}\left|1+\mathrm{e}^{i \vartheta}\right|^{2 t} \mathrm{~d} \vartheta=\frac{2^{2 t} \Gamma(t+1 / 2)}{\sqrt{\pi} \Gamma(t+1)} \quad(t>0),
$$

et notons d'emblée, à fins de référence ultérieure, que $\lambda(t) \leqslant 2^{2 t-1}$ pour tout $t \geqslant 1$ avec égalité si, et seulement si, $t=1$.

Théorème 1.3. Soient $A>0, c>0, t \geqslant 1, \eta \in] 0,1[$ et $g$ une fonction multiplicative à valeurs positives ou nulles, vérifiant (1.1), (1.2) et (1.3). Il existe une constante $\alpha=\alpha(g ; c, t, \eta)>0$ telle que l'on ait

$$
\begin{aligned}
\mathfrak{S}_{t}(x, \mu ; g) & :=\sum_{n \leqslant x} g(n) \Delta(n, \mu)^{2 t} \\
& \ll x \mathrm{e}^{\alpha \sqrt{\log _{2} x \log _{3} x}}(\log x)^{y-1+(\lambda(t) y-y-t)^{+}} \quad(x \geqslant 16) .
\end{aligned}
$$

Le facteur $\log _{3} x$ peut être omis dans le membre de droite lorsque $y>t /(\lambda(t)-1)$.

2. Voir $[20]$ pour une amélioration de l'exposant $2 \sqrt{t}$. 
Ainsi, la relation (1.11) est également valable en remplaçant $\chi$ par $\mu$. Au vu de ces résultats, il est intéressant de déterminer si les quantités

$$
\frac{1}{x} \sum_{n \leqslant x} \mu(n)^{2} \Delta(n, \chi)^{2}, \quad \frac{1}{x} \sum_{n \leqslant x} \mu(n)^{2} \Delta(n, \mu)^{2}
$$

tendent ou non vers l'infini avec $x$. La réponse est positive : Maier a en effet établi dans [12] que l'on a, pour presque tout entier $n$,

$$
\Delta(n, \mu)>\left(\log _{2} n\right)^{\gamma_{0}+o(1)}
$$

avec

$$
\gamma_{0}:=(\log 2) /|\log (1-1 / \log 3)| \approx 0,28754
$$

et sa démonstration fournit la même borne inférieure dans le cas d'un caractère réel. Nous pouvons donc énoncer le résultat suivant.

Théorème 1.4. Nous avons

$$
\sum_{n \leqslant x} \mu(n)^{2} \Delta(n, \mu)^{2}>x\left(\log _{2} x\right)^{2 \gamma_{0}+o(1)} \quad(x \rightarrow \infty)
$$

où $\gamma_{0}$ est la constante définie en (1·17). La même relation vaut pour $\Delta(n, \chi)$.

Notons que l'inégalité $(1 \cdot 16)$, a fortiori valable pour $\Delta(n)$, a été récemment améliorée dans ce cas par Maier et Tenenbaum [14], qui établissent

$$
\Delta(n)>\left(\log _{2} n\right)^{\gamma_{1}+o(1)}
$$

pour presque tout entier $n$, avec

$$
\gamma_{1}:=(\log 2) / \log \left(\frac{1-1 / \log 27}{1-1 / \log 3}\right) \approx 0,33827 .
$$

Bien que les complications techniques soient significatives, il est très vraisemblable que la méthode de [12] puisse être intégrée à celle de [14] pour permettre de remplacer $\gamma_{0}$ par $\gamma_{1}$ dans (1.16) et dans le Théorème 1.4.

Lorsque $t>1$, nous pouvons minorer le moment d'ordre $2 t$ de $\Delta(n, \mu)$ par une puissance de logarithme strictement positive.

Théorème 1.5. Soient $y>0, t \geqslant 1$. Nous avons

$$
\sum_{n \leqslant x} \mu(n)^{2} y^{\omega(n)} \Delta(n, \mu)^{2 t} \gg x(\log x)^{y-1+\left(2^{t} y-y-t\right)^{+}} \quad(x \rightarrow \infty) .
$$

Notons, cependant, que nous ne disposons pas de renforcement de ce dernier résultat comparable à $(1 \cdot 10)$ pour les grandes valeurs de $t$.

\subsection{Applications}

Étant donnée une forme binaire $P \in \mathbb{Z}[X, Y]$ de degré 4 et de discriminant non nul, tout modèle propre et lisse $V$ de la surface affine

$$
y^{2}+z^{2}=P(x, 1)
$$

est une surface de Châtelet sur $\mathbb{Q}$. La conjecture de Manin propose un équivalent asymptotique pour le nombre $N_{P}(B)$ des points $\mathbb{Q}$-rationnels de la variété $V$ dont la hauteur n'excède pas une borne déterminée $B$. Elle a donné lieu ces dernières années à de nombreux travaux, 
notamment [16]. Considéré spécifiquement depuis 2005, le cas particulier des surfaces de Châtelet a donné lieu à une confirmation de la conjecture sous certaines conditions de factorisation pour la forme $P$ - cf. notamment [2] et [3].

Désignons par $r(n)$ le nombre de décompositions d'un entier générique $n$ comme somme de deux carrés et, pour toute une forme binaire $P \in \mathbb{Z}[X, Y]$ de degré 4 , introduisons la quantité

$$
Q_{P}(\xi):=\sum_{\boldsymbol{x} \in \mathbb{Z}^{2} \cap \mathcal{R}_{P}(\xi)} r(P(\boldsymbol{x}))
$$

où l'on a posé

$$
\mathcal{R}_{P}(\xi):=\left\{\boldsymbol{x} \in \mathbb{R}^{2}:\|\boldsymbol{x}\|_{\infty} \leqslant \xi, P(\boldsymbol{x})>0\right\} \quad(\xi>0) .
$$

L'évaluation asymptotique de $N_{P}(B)$ peut être reformulée en termes d'estimations de quantités plus générales, mais essentiellement de même nature que $Q_{P}(\xi)$, sous réserve d'une uniformité suffisante en divers paramètres, notamment les coefficients de la forme $P$.

Tant pour $N_{P}(B)$ que pour $Q_{P}(\xi)$ et ses avatars, la forme du résultat asymptotique conjecturé et les méthodes nécessaires pour l'obtenir sont subordonnées au type de factorisation de $P$ dans $\mathbb{Q}$ et à la nature des facteurs dans $\mathbb{Q}[i]$. La liste suivante explicite tous les cas possibles :

(i) $P=L_{1} L_{2} L_{3} L_{4}$, où les $L_{j}$ sont des formes linéaires de $\mathbb{Q}[X]$;

(ii) $P=L_{1} L_{2} Q$ où les $L_{j}$ sont des formes linéaires, et $Q$ une forme quadratique irréductible sur $\mathbb{Q}$ mais réductible sur $\mathbb{Q}[i]$;

(iii) $P=L_{1} L_{2} Q$ où les $L_{j}$ sont des formes linéaires, et $Q$ une forme quadratique irréductible sur $\mathbb{Q}[i]$;

(iv) $P=L C$, où $L$ est une forme linéaire, et $C$ est une forme cubique irréductible $\operatorname{sur} \mathbb{Q}$;

(v) $P=Q_{1} Q_{2}$, où les $Q_{j}$ sont formes quadratiques irréductibles sur $\mathbb{Q}$, mais dont l'une au moins est réductible sur $\mathbb{Q}[i]$;

(vi) $P=Q_{1} Q_{2}$ où les $Q_{j}$ sont formes quadratiques irréductibles sur $\mathbb{Q}[i]$;

(vii) $P$ est irréductible sur $\mathbb{Q}$, mais est réductible sur $\mathbb{Q}[i]$;

(viii) $P$ est une forme irréductible sur $\mathbb{Q}[i]$.

Le premier exemple d'une formule asymptotique pour $Q_{P}(\xi)$ lorsque $\xi \rightarrow \infty$ a été obtenu par Daniel [7] pour le polynôme $P(X, Y)=X^{4}+Y^{4}$, qui est du type (vii). À la suite de ce travail, d'autres progrès ont été accomplis.

Pour les formes de type (i), l'évaluation asymptotique de $Q_{P}(\xi)$ a été traitée par HeathBrown dans [10]. Les précisions nécessaires à l'approche de la conjecture de Manin ont été obtenues dans [1], ce qui a effectivement permis l'estimation asymptotique de $N_{P}(B)$ dans [3].

Les mêmes méthodes fonctionnent pour le type (iv) : les évaluations de $Q_{P}(\xi)$ et de $N_{P}(B)$ ont ainsi été obtenues dans [2]. Cette approche permet encore de disposer des autres cas dans lesquels $P$ est divisible par une forme linéaire, autrement dit les types (ii) et (iii).

Quoiqu'ils n'aient pas été explicitement traités dans la littérature, les types (v) et (vii), dans lesquels l'un au moins des facteurs irréductibles de $P$ sur $\mathbb{Q}$ est réductible sur $\mathbb{Q}[i]$ relèvent des techniques précédentes.

Cependant, les types (vi) et (viii) ne se réduisent pas à ces approches antérieures et des idées nouvelles sont nécessaires pour les aborder. Le Théorème 1.1 (spécialisé avec $t=1$, $y=1$ ) est la clef de voûte des estimations asymptotiques obtenues dans [5] pour les quantités du type $Q_{P}(\xi)$, qui nous ont à leur tour permis d'établir la conjecture de Manin dans ces hypothèses.

À titre d'exemple, nous pouvons ainsi annoncer le résultat suivant.

Théorème 1.6 [5]. Soit $P \in \mathbb{Z}[X, Y]$ une forme binaire de degré 4 , irréductible sur $\mathbb{Q}[i]$ ou produit de deux formes quadratiques linéairement indépendantes et irréductibles sur $\mathbb{Q}[i]$. Il existe une constante $K_{P}$ telle que l'on ait

$$
Q_{P}(\xi)=K_{P} \xi^{2}+O\left(\frac{\xi^{2}}{(\log \xi)^{1 / 48}}\right) \quad(\xi \rightarrow \infty) .
$$


Le calcul de la constante $K_{P}$ est explicité dans [5], où l'on établit en fait une version uniforme en fonction des coefficients de $P$.

Il est à noter que, dans le cas des factorisations de type (v) et (vii), on s'attend à une relation asymptotique de la forme

$$
\mathcal{Q}_{P}(\xi) \sim K_{P} \xi^{2}(\log \xi)^{r} \quad(\xi \rightarrow \infty),
$$

où $r$ est le nombre de facteurs de $P$ qui sont irréductibles sur $\mathbb{Q}$ mais réductibles sur $\mathbb{Q}[i]$. L'évaluation asymptotique de $N_{P}(B)$ est en fait favorisée par la présence de tels facteurs: dès que $r \geqslant 1$, les estimations en moyenne de $\Delta(\cdot, \chi)$ ne sont plus nécessaires pour évaluer $\mathcal{Q}_{P}(\xi)$ et confirmer la conjecture de Manin.

On a classiquement $r=4\left(\mathbf{1} * \chi_{4}\right)$, où $\chi_{4}$ désigne le caractère de Dirichlet non principal de module 4. La démonstration du Théorème 1.6 fonctionne, mutatis mutandis, pour toute fonction arithmétique du type $\mathbf{1} * \chi$ où $\chi$ est un caractère de Dirichlet réel non principal. Cependant, ces résultats s'appuyent sur un théorème de type Bombieri-Vinogradov pour la répartition des ensembles $\mathcal{R}(\xi)$ dans les classes de congruence $P(\boldsymbol{x}) \equiv 0(\bmod d)$ qui ne possède pas de généralisation suffisante pour étendre le Théorème 1.6 à une plus vaste classe de fonctions arithmétiques.

Comme annoncé plus haut, nous avons pu confirmer la conjecture de Manin en nous appuyant sur des estimations uniformes de même nature que (1·19). Ainsi, pour les familles de variétés correspondant aux types (vi) et (vii), nous établissons dans [5] l'existence d'une constante $C_{P}$ telle que

$$
N_{P}(B)=\left\{C_{P}+O\left((\log B)^{-1 / 100}\right)\right\} B \log B \quad(B \rightarrow \infty) .
$$

Remerciements. Les auteurs prennent plaisir à remercier ici Helmut Maier pour son aide lors de l'élaboration de cet article, notamment la vérification que sa preuve du résultat principal de [12] s'adapte sans difficulté au cas d'un caractère de Dirichlet réel et non principal.

\section{Lemmes}

Définissons

$$
E(n):=\min _{d d^{\prime} \mid n, d<d^{\prime}} \log \left(d^{\prime} / d\right), \quad E^{*}(n):=\min \{1, E(n)\}
$$

et posons

$$
M_{q}(n, \chi):=\int_{0}^{1} \int_{\mathbb{R}} \Delta(n, \chi ; u, v)^{q} \mathrm{~d} u \mathrm{~d} v \quad(n \geqslant 1) .
$$

Observons d'emblée que

$$
M_{2 q}(n, \chi) \geqslant \log 2-\frac{1}{2}(\log 2)^{2} \geqslant \frac{1}{4} \quad(n \geqslant 1, q \geqslant 1) .
$$

Cette minoration triviale est obtenue en considérant la contribution des couples $(u, v)$ satisfaisant $u<0$ et $-u<v<\min \{\log 2-u, 1\}$, pour lesquels $\Delta(n, \chi ; u, v)=1$.

Nous considérerons également les quantités

$$
M_{q}(n):=\int_{\mathbb{R}} \Delta(n ; u, 1)^{q} \mathrm{~d} u, \quad M_{q}^{*}(n)=\sum_{\begin{array}{c}
d_{1}, \ldots, d_{q} \mid n \\
\max d_{r} \leqslant \min d_{r}
\end{array}} 1 \quad(n \geqslant 1) .
$$

D'après [13], on a

$$
M_{q}^{*}(n) \leqslant 2^{q} M_{q}(n) \quad(q \geqslant 1, n \geqslant 1) .
$$


Rappelons également la majoration établie dans [18]

$$
M_{j}(n) \leqslant M_{q}(n)^{(j-1) /(q-1)} \tau(n)^{(q-j) /(q-1)} \quad(n \geqslant 1, q \geqslant 2,1 \leqslant j \leqslant q-1) .
$$

Lemme 2.1. On a

$$
\Delta(n, \chi)^{2} \leqslant 2^{5}+2^{3+2 / q} E^{*}(n)^{-2 / q} M_{2 q}(n, \chi)^{1 / q} \quad(n>1, q \geqslant 1) .
$$

En particulier, si $0<\eta \leqslant 1, E^{*}(n) \geqslant \eta, 1 \leqslant q \leqslant r$, on a

$$
M_{2 r}(n, \chi)^{1 / r} \leqslant 2^{8-8 q / r} \eta^{2 / r-2 / q} M_{2 q}(n, \chi)^{1 / q} .
$$

Démonstration. L'inégalité $(2 \cdot 4)$ est trivialement satisfaite si $\Delta(n, \chi) \leqslant 4$.

Dans le cas contraire, considérons $u_{0}, v_{0}, 0<v_{0} \leqslant \frac{1}{2}$ tels que

$$
\left|\Delta\left(n, \chi ; u_{0}, v_{0}\right)\right| \geqslant \frac{1}{2} \Delta(n, \chi) .
$$

Soient $d$ le plus petit diviseur de $n$ dans $\left.] \mathrm{e}^{u_{0}}, \mathrm{e}^{u_{0}+v_{0}}\right]$ et $d^{\prime}$ le plus petit diviseur de $n$ excédant $\mathrm{e}^{u_{0}+v_{0}}$ — nous omettons le cas ou l'un ou l'autre de ces diviseurs n'existe pas : le raisonnement est similaire, et en fait plus simple. Pour $u_{0}<u \leqslant u_{0}+\frac{1}{2} E(n), v_{0}<v \leqslant v_{0}+\frac{1}{2} E(n)$, on a

$$
\begin{aligned}
|\Delta(n, \chi ; u, v)| & =\left|\Delta\left(n, \chi ; u_{0}, v_{0}\right)-\mathbf{1}_{] u_{0}, u\right]}(\log d) \chi(d)-\mathbf{1}_{] u_{0}+v_{0}, u+v\right]}\left(\log d^{\prime}\right) \chi\left(d^{\prime}\right)\right| \\
& \geqslant \frac{1}{2} \Delta(n, \chi)-2 .
\end{aligned}
$$

Il suit

$$
E^{*}(n)^{2}\{\Delta(n, \chi)-4\}^{2 q} \leqslant 2^{2+2 q} M_{2 q}(n, \chi) .
$$

Cela implique immédiatement $(2 \cdot 4)$ en notant que

$$
\Delta(n, \chi)^{2} \leqslant 2 \cdot 4^{2}+2\{\Delta(n, \chi)-4\}^{2} .
$$

Pour montrer $(2 \cdot 5)$, nous observons que l'on a, sous les hypothèses indiquées,

$$
1 \leqslant(2 / \eta)^{2 / q} M_{2 q}(n)^{1 / q} \leqslant 2^{2} \eta^{-2 / q} M_{2 q}(n)^{1 / q}
$$

et donc

$$
\begin{aligned}
M_{2 r}(n, \chi) & \leqslant \Delta(n, \chi)^{2(r-q)} M_{2 q}(n, \chi) \\
& \leqslant\left\{2^{8} \eta^{-2 / q} M_{2 q}(n, \chi)^{1 / q}\right\}^{r-q} M_{2 q}(n, \chi) \leqslant 2^{8 r-8 q} \eta^{2-2 r / q} M_{2 q}(n, \chi)^{r / q} .
\end{aligned}
$$

À fins de référence ultérieure, posons

$$
\mathcal{G}(\sigma):=\sum_{n \geqslant 1} \frac{\mu(n)^{2} g(n)}{n^{1+\sigma}},
$$

de sorte que nous avons, en vertu de $(1 \cdot 3)$,

$$
\mathcal{G}(\sigma) \asymp \sigma^{-y} .
$$

Nous notons $P^{+}(n)$ le plus grand facteur premier d'un entier générique $n$ avec la convention $P^{+}(1)=1$. Nous désignons également par $\left\{p_{k}(n)\right\}_{k=1}^{\omega(n)}$ la suite croissante des facteurs premiers distincts d'un entier générique $n$ et posons, dans ce paragraphe et dans la section 3.1,

$$
n_{k}:= \begin{cases}\prod_{1 \leqslant j \leqslant k} p_{j}(n) & \text { si } \omega(n) \geqslant k, \\ n & \text { si } \omega(n)<k .\end{cases}
$$


Lemme 2.2. Soient $A>0 c>0, t \geqslant 1, \eta \in] 0,1\left[\right.$, et $g \in \mathcal{M}_{A}(c, \eta)$ une fonction arithmétique telle que $y=y(g)>0$.

(i) Si $0<y \leqslant 1$, nous avons, uniformément sous les conditions $0<\sigma \leqslant \frac{1}{2}, k \geqslant 1$, $\mathcal{A}_{k}:=\left\{n \in \mathbb{N}^{*}: E\left(n_{k}\right)>(420)^{-k t / y}\right\}$,

$$
R_{k}(\sigma):=\sum_{n \in \mathbb{N}^{*} \backslash \mathcal{A}_{k}} \frac{\mu(n)^{2} g(n)}{n^{1+\sigma}} \ll \frac{\mathrm{e}^{-16 k t / 9}}{\sigma^{y}} .
$$

(ii) Pour tout $y>0$, nous avons, uniformément pour $\sigma>0$,

$$
\sum_{\substack{n \geqslant 1 \\ E(n) \leqslant \sigma^{3 \cdot 4^{t} y}}} \frac{\mu(n)^{2} g(n) \tau(n)^{2 t}}{n^{1+\sigma}} \ll 1 .
$$

Démonstration. Commençons par établir le point (i). Désignons par $\omega_{k}(n)$ le nombre des facteurs premiers d'un entier générique $n$ qui n'excèdent pas $u_{k}:=\exp \left(70^{k t / y} / k\right)$. Étant donné un paramètre $v \in] 0,1$, nous pouvons majorer la contribution à $R_{k}(\sigma)$ de l'ensemble des entiers $n$ tels que $n_{k}>u_{k}^{k}$ par

$$
\sum_{n \geqslant 1} \frac{g(n) v^{\omega_{k}(n)-k}}{n^{1+\sigma}} \ll_{v} \frac{v^{-k} 70^{k t(v-1)} k^{y(1-v)}}{\sigma^{y}} \ll \frac{\mathrm{e}^{-16 k t / 9}}{\sigma^{y}},
$$

pour le choix $v:=1 /(t \log 70)$. La contribution complémentaire ne dépasse pas

$$
\begin{aligned}
& \sum_{\substack{1 \leqslant d, d^{\prime} \leqslant \exp \left(70^{k t / y}\right) \\
0<\left|\log \left(d^{\prime} / d\right)\right| \leqslant(420)^{-k t / y} \\
m \geqslant 1}} \frac{\mu\left(m d d^{\prime}\right)^{2} g\left(m d d^{\prime}\right)}{\left(d d^{\prime} m\right)^{1+\sigma}} \\
& \ll \mathcal{G}(\sigma) \sum_{420^{k t / y}-1 \leqslant d \leqslant \exp \left(70^{k t / y}\right)} \frac{\mu(d)^{2} g(d)}{d} \sum_{0<\left|\log \left(d^{\prime} / d\right)\right| \leqslant(415)^{-k t / y}} \frac{\mu\left(d^{\prime}\right)^{2} g\left(d^{\prime}\right)}{d^{\prime}} .
\end{aligned}
$$

La somme intérieure relève du théorème de Shiu [17] : elle est $\ll 415^{-k t / y}(\log d)^{y-1}$. Nous obtenons donc la majoration globale de la somme triple

$$
\ll \frac{415^{-k t / y}}{\sigma^{y}} \sum_{(420)^{k t / y}-1 \leqslant d \leqslant \exp (70)^{k t / y}} \frac{\mu(d)^{2} g(d)(\log d)^{y-1}}{d} \ll \frac{14^{k t}}{83^{k t} \sigma^{y}},
$$

où nous avons utilisé une sommation d'Abel en majorant en moyenne $g(d)(\log d)^{y-1}$ par $\ll(\log d)^{y-1}$. Comme $\log \frac{83}{14}>\frac{16}{9}$, nous obtenons bien la majoration requise.

Pour établir la majoration $(2 \cdot 8)$, nous posons $Y:=3 \cdot 4^{t} y$ et nous écrivons

$$
\begin{aligned}
\sum_{\substack{n \geqslant 1 \\
E(n) \leqslant \sigma^{Y}}} \frac{\mu(n)^{2} g(n) \tau(n)^{2 t}}{n^{1+\sigma}} & \leqslant \sum_{\substack{m, d, d^{\prime} \\
1 / 2 \sigma^{Y} \leqslant d<d^{\prime} \leqslant\left(1+\sigma^{Y}\right) d}} \frac{g(m) g(d) g\left(d^{\prime}\right) \tau(m)^{2 t} \tau(d)^{2 t} \tau\left(d^{\prime}\right)^{2 t}}{\left(m d d^{\prime}\right)^{1+\sigma}} \\
& \ll \frac{1}{\sigma^{4^{t} y}} \sum_{1 / 2 \sigma^{Y} \leqslant d<d^{\prime} \leqslant\left(1+\sigma^{Y-1}\right) d} \frac{g(d) g\left(d^{\prime}\right) \tau(d)^{2 t} \tau\left(d^{\prime}\right)^{2 t}}{\left(d d^{\prime}\right)^{1+\sigma}} \\
& \ll \sigma^{Y-1-4^{t} y} \sum_{d} \frac{g(d) \tau(d)^{2 t}(\log d)^{4^{t} y-1}}{d^{1+\sigma}} \ll \sigma^{Y-3 \cdot 4^{t} y} \ll 1,
\end{aligned}
$$

où nous avons de nouveau fait appel au théorème de Shiu [17]. 
Posons $\tau(n, \chi ; \vartheta):=\sum_{d \mid n} \chi(d) d^{i \vartheta}(n \geqslant 1, \vartheta \in \mathbb{R})$ et

$$
\tau^{*}(n, \chi):=\frac{2}{\pi} \int_{\mathbb{R}} \frac{|\tau(n, \chi ; \vartheta)|^{2}}{6+\vartheta^{2}} \mathrm{~d} \vartheta \quad(n \geqslant 1) .
$$

Lemme 2.3. Pour $n \geqslant 1$, on a

$$
M_{2}(n, \chi) \leqslant \tau^{*}(n, \chi) .
$$

Démonstration. Pour tous $u, v$ sauf au plus un nombre fini, on a

$$
\Delta(n, \chi ; u, v)=\frac{1}{2 \pi i} \int_{\mathbb{R}} \frac{1-\mathrm{e}^{-i \vartheta v}}{\vartheta} \mathrm{e}^{-i u \vartheta} \tau(n, \chi ; \vartheta) \mathrm{d} \vartheta .
$$

La formule de Plancherel implique donc

$$
\int_{\mathbb{R}} \Delta(n, \chi ; u, v)^{2} \mathrm{~d} u=\frac{2}{\pi} \int_{\mathbb{R}} \frac{(\sin \vartheta v / 2)^{2}|\tau(n, \chi ; \vartheta)|^{2}}{\vartheta^{2}} \mathrm{~d} \vartheta
$$

d'où

$$
M_{2}(n, \chi)=\frac{1}{\pi} \int_{\mathbb{R}}\left(1-\frac{\sin \vartheta}{\vartheta}\right) \frac{|\tau(n, \chi ; \vartheta)|^{2}}{\vartheta^{2}} \mathrm{~d} \vartheta .
$$

Or

$$
1-\frac{\sin \vartheta}{\vartheta} \leqslant \frac{2 \vartheta^{2}}{6+\vartheta^{2}} \quad(\vartheta \in \mathbb{R})
$$

Nous observons à fins de référence ultérieure que ce qui précède implique

$$
\int_{\mathbb{R}} \Delta(n, \chi ; u, v)^{2} \mathrm{~d} u \ll \int_{\mathbb{R}} \frac{|\tau(n, \chi ; \vartheta)| v^{2}}{1+\vartheta^{2} v^{2}} \mathrm{~d} \vartheta \ll \int_{\mathbb{R}} \frac{|\tau(n, \chi ; \vartheta)|}{1+\vartheta^{2}} \mathrm{~d} \vartheta \ll M_{2}(n, \chi) \quad(0 \leqslant v \leqslant 1) .
$$

Pour $q \geqslant 1,0 \leqslant j \leqslant q$, nous introduisons les quantités

$$
N_{j, q}(n, w):=\int_{0}^{1} \int_{\mathbb{R}} \Delta(n, \chi ; u, v)^{j} \Delta(n, \chi ; u-w, v)^{q-j} \mathrm{~d} u \mathrm{~d} v \quad(n \geqslant 1, w \in \mathbb{R}) .
$$

Lemme 2.4. Pour $1 \leqslant j \leqslant q-1, A>0, c>0, \eta \in] 0,1\left[, g \in \mathcal{M}_{A}(c, \eta), n \geqslant 1, x \geqslant 2\right.$, nous avons

$$
\sum_{p>x} \frac{N_{2 j, 2 q}(n, \log p) g(p) \log p}{p} \leqslant y M_{2 q}(n, \chi)^{(q-2) /(q-1)} \tau^{*}(n, \chi)^{q /(q-1)}+R_{q}(n, x)
$$

avec $y=y(g)$ et

$$
R_{q}(n, x) \ll \mathrm{e}^{-c(\log x)^{\eta}} 4^{q} M_{2 q}(n)^{(2 q-2) /(2 q-1)} \tau(n)^{2 q /(2 q-1)},
$$

où la constante implicite dépend au plus de $b, c$ et $\eta$.

Démonstration. Notant provisoirement $h:=2 q-2 j$, nous avons

$$
\begin{aligned}
S_{h}(n, x ; u, v) & :=\sum_{p>x} \frac{\Delta(n, \chi ; u-\log p, v)^{h} g(p) \log p}{p} \\
& =\sum_{d_{1}, \ldots, d_{h} \mid n} \chi\left(d_{1} \cdots d_{h}\right) \sum_{u-\log \min d_{r}<\log p \leqslant u+v-\log \max d_{r}} \frac{g(p) \log p}{p} .
\end{aligned}
$$


La somme intérieure est vide si $\log \left(\max d_{r} / \min d_{r}\right)>v$. Dans le cas contraire, on déduit de (1.3) par sommation d'Abel qu'elle vaut

$$
y \int_{u-\log \min d_{r}}^{u+v-\log \max d_{r}} \mathbf{1}_{] 0, \infty[}(t-\log x) \mathrm{d} t+O\left(\mathrm{e}^{-c(\log x)^{\eta}}\right) .
$$

D'où, par $(2 \cdot 2)$,

$$
\begin{aligned}
S_{h}(n, x ; u, v) & =y \int_{\log x}^{\infty} \Delta(n, \chi ; u-t, v)^{h} \mathrm{~d} t+O\left(\mathrm{e}^{-c(\log x)^{\eta}} M_{h}^{*}(n)\right) \\
& \leqslant y \int_{\mathbb{R}} \Delta(n, \chi ; t, v)^{h} \mathrm{~d} t+O\left(\mathrm{e}^{-c(\log x)^{\eta}} 2^{h} M_{h}(n)\right) .
\end{aligned}
$$

En reportant dans le membre de gauche de $(2 \cdot 12)$, nous obtenons

$$
\sum_{p>x} \frac{N_{2 j, 2 q}(n, \log p) g(p) \log p}{p} \leqslant y Q_{j}+O\left(\mathrm{e}^{-c(\log x)^{\eta}} 4^{q-j} M_{2 q-2 j}(n) M_{2 j}(n)\right)
$$

avec

$$
Q_{j}:=\int_{0}^{1}\left(\int_{\mathbb{R}} \Delta(n, \chi ; u, v)^{2 j} \mathrm{~d} u \int_{\mathbb{R}} \Delta(n, \chi ; t, v)^{2 q-2 j} \mathrm{~d} t\right) \mathrm{d} v .
$$

Or, d'après l'inégalité de Hölder avec exposants $(q-1) /(j-1),(q-1) /(q-j)$ utilisée, lorsque $j \geqslant 2$, avec la décomposition

$$
\Delta(n, \chi ; u, v)^{2 j}=\Delta(n, \chi ; u, v)^{2 q(j-1) /(q-1)} \Delta(n, \chi ; u, v)^{2(q-j) /(q-1)},
$$

nous avons

$$
\begin{aligned}
\int_{\mathbb{R}} \Delta(n, \chi ; u, v)^{2 j} \mathrm{~d} u & \\
& \leqslant\left(\int_{\mathbb{R}} \Delta(n, \chi ; u, v)^{2 q} \mathrm{~d} u\right)^{(j-1) /(q-1)}\left(\int_{\mathbb{R}} \Delta(n, \chi ; u, v)^{2} \mathrm{~d} u\right)^{(q-j) /(q-1)} .
\end{aligned}
$$

Appliquant cela pour les exposants $j$ et pour $q-j$, nous obtenons

$$
\begin{aligned}
Q_{j} & \leqslant \int_{0}^{1} \mathrm{~d} v\left(\int_{\mathbb{R}} \Delta(n, \chi ; u, v)^{2 q} \mathrm{~d} u\right)^{(q-2) /(q-1)}\left(\int_{\mathbb{R}} \Delta(n, \chi ; u, v)^{2} \mathrm{~d} u\right)^{q /(q-1)} \\
& \ll M_{2 q}(n, \chi)^{(q-2) /(q-1)} M_{2}(n, \chi)^{q /(q-1)},
\end{aligned}
$$

compte tenu de la remarque qui suit le Lemme 2.3. Le résultat annoncé découle de cette majoration grâce à $(2 \cdot 9)$ et $(2 \cdot 3)$.

Lemme 2.5. Soient $A>0, c>0, \eta \in] 0,1[, t \geqslant 1, \chi$ un caractère de Dirichlet réel non principal, et $g \in \mathcal{M}_{A}(\chi ; c, \eta)$. Nous avons, uniformément pour $0<|\vartheta| \leqslant 1$,

$$
\begin{aligned}
& \sum_{p \leqslant x} \frac{g(p)\left|1+\chi(p) p^{i \vartheta}\right|^{2 t}}{p} \\
& \quad=y \lambda(t) \log (1+|\vartheta| \log x)+(y+z) 2^{2 t-1} \log \left(\frac{\log x}{1+|\vartheta| \log x}\right)+O(1)
\end{aligned}
$$

et, uniformément pour $|\vartheta|>1$,

$$
\sum_{p \leqslant x} \frac{g(p)\left|1+\chi(p) p^{i \vartheta}\right|^{2 t}}{p} \leqslant y \lambda(t) \log _{2} x+B(t ; c, \eta) \log _{2}(2+|\vartheta|),
$$

où $B(t ; c, \eta)$ est une constante convenable.

Démonstration. Observons d'emblée que la relation

$$
\sum_{p \leqslant x} \frac{g(p)}{p}=y \log _{2} x+O(1) \quad(x \geqslant 3),
$$

où $y=y(g)$, est une conséquence immédiate de $(1 \cdot 3)$. 
Le membre de gauche de $(2 \cdot 13)$ vaut

$$
\sum_{p \leqslant x} \frac{1+\chi(p)}{2}\left|1+p^{i \vartheta}\right|^{2 t} \frac{g(p)}{p}+\sum_{p \leqslant x} \frac{1-\chi(p)}{2}\left|1-p^{i \vartheta}\right|^{2 t} \frac{g(p)}{p} .
$$

Une intégration par parties semblable à celle du lemme III.4.13 de [21] et dont nous omettons les détails permet de déduire de (1.3) et (1.4) que l'on a, uniformément pour $\vartheta \neq 0$,

$$
\begin{aligned}
\sum_{w<p \leqslant x} \frac{1 \pm \chi(p)}{2} \mid & 1 \pm\left. p^{i \vartheta}\right|^{2 t} \frac{g(p)}{p} \\
= & \frac{(y \pm z) \lambda(t)}{2} \log \left(\frac{\log x}{\log w}\right)+O\left(\frac{1}{|\vartheta| \log w}+\frac{1+|\vartheta|}{\mathrm{e}^{(c / 2)(\log w)^{\eta}}}\right) .
\end{aligned}
$$

Supposons $1 / \log x<|\vartheta| \leqslant 1$. En choisissant $w:=\exp \{(\log x) /(1+|\vartheta| \log x)\}$ et en approchant $\left|1 \pm p^{i \vartheta}\right|^{2 t}$ par $|1 \pm 1|^{2 t}$ pour $p \leqslant w$, on en déduit immédiatement la relation (2·13). Le même résultat est valable lorsque $|\vartheta| \leqslant 1 / \log x$, en choisissant à présent $w:=x$.

Lorsque $1<|\vartheta| \leqslant \exp \left\{(c / 2)(\log x)^{\eta}\right\}-2$, nous appliquons (2.16) avec

$$
w:=\exp \left(\{(2 / c) \log (1+|\vartheta|)\}^{1 / \eta}\right),
$$

nous utilisons la majoration triviale $g(p)\left|1+\chi(p) p^{i \vartheta}\right|^{2 t} \ll g(p)$ pour $p \leqslant w$ et nous appliquons $(2 \cdot 15)$. Cela fournit bien $(2 \cdot 14)$.

Enfin, lorsque $|\vartheta|+2>\exp \left\{(c / 2)(\log x)^{\eta}\right\}$, nous nous contentons d'observer que le membre de gauche de $(2 \cdot 14)$ est $\ll \log _{2} x \ll \log _{2}(2+|\vartheta|)$.

Quelques notations sont nécessaires pour l'énoncé du lemme suivant. Étant donné un entier $t \geqslant 1$, nous désignons par $\left\{e_{j}: 1 \leqslant j \leqslant t\right\}$ la base canonique de $\mathbb{R}^{t}$ et par $\mathcal{W}_{t}$ l'ensemble des formes linéaires $w \in \mathcal{L}^{*}\left(\mathbb{R}^{t}\right):=\mathcal{L}\left(\mathbb{R}^{t}, \mathbb{R}\right)$ telles que $w\left(e_{j}\right) \in\{-1,0,1\}$ pour tout $j$. Lorsque $w \in \mathcal{W}_{t}$, nous posons $|w|:=\sum_{1 \leqslant j \leqslant t}\left|w\left(e_{j}\right)\right|$. Nous introduisons alors la famille d'intégrales

$$
I_{y}(\delta, X)=\int_{\mathbb{R}^{t}} \prod_{\substack{w \in \mathcal{W}_{t} \\|w| \equiv 0(\bmod 2)}}\left(1+\frac{X}{1+|w(\boldsymbol{\vartheta})|}\right)^{2^{t-|w|} y} \prod_{1 \leqslant j \leqslant t} \frac{\mathrm{d} \vartheta_{j}}{1+\left(\vartheta_{j} / X\right)^{\delta}},
$$

où $\delta, X, y$ sont les paramètres vérifiant $\delta>1, X \geqslant 1, y>0$. Notons, à fins de référence ultérieure, que le produit en $w$ est $\ll X^{2^{2 t-1} y}$ uniformément en $\vartheta$, puisque

$$
\sum_{\substack{0 \leqslant m \leqslant t \\
2 \mid m}} 2^{m}\left(\begin{array}{c}
t \\
m
\end{array}\right) 2^{t-m} y=2^{t-1} y \sum_{0 \leqslant m \leqslant t}\left\{1+(-1)^{m}\right\}\left(\begin{array}{c}
t \\
m
\end{array}\right)=2^{2 t-1} y .
$$

Lemme 2.6. Pour tous $\delta>1, t \in \mathbb{N}, t \geqslant 2, y>2^{1-t}$, et uniformément pour $X \geqslant 1$, nous avons

$$
I_{y}(\delta, X) \ll X^{2^{2 t-1} y} .
$$

Démonstration. Étant donnée une famille libre $U:=\left(u_{1}, \ldots, u_{j}\right)$ de $j$ formes linéaires de $\mathcal{W}_{t}$, nous introduisons

$$
V(U, X):=\left\{\boldsymbol{\vartheta} \in \mathbb{R}^{t}: \max _{1 \leqslant h \leqslant j}\left|u_{h}(\boldsymbol{\vartheta})\right| \leqslant X,\left(\forall w \in \mathcal{W}_{t}\right)|w(\boldsymbol{\vartheta})| \leqslant X \Rightarrow w \in \operatorname{Vect}(U)\right\} .
$$

Lorsque $j=0$, nous posons

$$
V(\varnothing, X):=\left\{\boldsymbol{\vartheta} \in \mathbb{R}^{t}:\left(\forall w \in \mathcal{W}_{t}\right)|w(\boldsymbol{\vartheta})|>X\right\} .
$$


Notant $\operatorname{rg}(U)$ le rang d'une famille de vecteurs de $\mathcal{W}_{t}$, nous avons clairement

$$
\mathbb{R}^{t}=\bigcup_{0 \leqslant j \leqslant t} \bigcup_{\substack{U \in \mathcal{W}_{t}^{j} \\ \operatorname{rg}(U)=j}} V(U, X)
$$

d'où

$$
I_{y}(\delta, X) \leqslant \sum_{0 \leqslant j \leqslant t} \sum_{\substack{U \in \mathcal{W}_{t}^{j} \\ \operatorname{rg}(U)=j}} J_{y}(X ; U)
$$

où $J_{y}(X ; U)$ désigne la contribution du domaine $V(X U)$ à l'intégrale $I_{y}(\delta, X)$.

Lorsque $\operatorname{rg}(U)=t$, nous avons

$$
J_{y}(X ; U) \ll X^{2^{2 t-1} y} \int_{V(U, X)} \prod_{\substack{w \in \mathcal{W}_{t} \\|w| \equiv 0(\bmod 2)}}\left(\frac{1}{1+|w(\vartheta)|}\right)^{2^{t-|w|} y} \mathrm{~d} \vartheta_{1} \cdots \mathrm{d} \vartheta_{t} \ll X^{2^{2 t-1} y}
$$

puisque l'intégrale ci-dessus est convergente dès que $2^{t-1} y>1$ : il suffit de restreindre le produit aux formes $w(\boldsymbol{\vartheta})= \pm\left(\vartheta_{h}+\vartheta_{1}\right)(2 \leqslant h \leqslant t), \pm\left(\vartheta_{2}-\vartheta_{1}\right)$.

Lorsque $j=\operatorname{rg}(U) \leqslant t-1$, nous complétons la famille $U$ en une base de $\mathcal{L}^{*}\left(\mathbb{R}^{t}\right)$ en choisissant des vecteurs $e_{h}^{*}(j<h \leqslant t)$ dans la base duale de la base canonique de $\mathbb{R}^{t}$, éventuellement réarrangés. On a alors $e_{h}^{*} \in \operatorname{Vect}(U)$ pour tout $h \in[1, j]$. Ainsi

$$
\begin{aligned}
J_{y}(X ; U) & \ll \int_{V(U, X)} \prod_{\substack{w \in \mathcal{W}_{t} \cap \operatorname{Vect}(U) \\
|w| \equiv 0(\bmod 2)}}\left(\frac{X}{1+|w(\boldsymbol{\vartheta})|}\right)^{2^{t-|w|} y} \frac{\mathrm{d} \vartheta_{1} \cdots \mathrm{d} \vartheta_{t}}{\prod_{j<h \leqslant t}\left\{1+\left(\vartheta_{h} / X\right)^{\delta}\right\}} \\
& \ll X^{\sigma(U)} \int_{V(U, X)} \prod_{\substack{w \in \mathcal{W}_{t} \cap \operatorname{Vect}(U) \\
|w|=2}}\left(\frac{1}{1+|w(\boldsymbol{\vartheta})|}\right)^{2^{t-2} y} \frac{\mathrm{d} \vartheta_{1} \cdots \mathrm{d} \vartheta_{t}}{\prod_{j<h \leqslant t}\left(1+\vartheta_{h}^{\delta}\right)} \ll X^{\sigma(U)+j}
\end{aligned}
$$

avec

$$
\sigma(U):=y \sum_{\substack{0 \leqslant m \leqslant t \\ 2 \mid m}} \sum_{\substack{w \in \mathcal{W} \cap \operatorname{Vect}(U) \\|w|=m}} 2^{t-m}+t-j,
$$

où l'ultime majoration est obtenue via le changement de variable

$$
\boldsymbol{\vartheta} \mapsto\left(u_{1}(\boldsymbol{\vartheta}), \ldots, u_{j}(\boldsymbol{\vartheta}), \vartheta_{j+1}, \ldots, \vartheta_{t}\right),
$$

qui permet de majorer la dernière intégrale par

$$
\ll \int_{\left(u_{1}, \ldots, u_{j}\right) \in[-X, X]^{j}} \mathrm{~d} u_{1} \cdots \mathrm{d} u_{j} \int_{\mathbb{R}^{t-j}} \frac{\mathrm{d} \vartheta_{j+1} \cdots \mathrm{d} \vartheta_{t}}{\prod_{j<h \leqslant t}\left(1+\vartheta_{h}^{\delta}\right)} \ll X^{j} .
$$

Il reste donc à majorer $\sigma(U)$.

Lorsque $j=\operatorname{rg}(U)=0$, on a $\sigma(U)=y 2^{t}+t \leqslant 2^{2 t-1} y$ puisque $t+2 \leqslant 2^{t}$ lorsque $t \geqslant 2$.

Lorsque $j=1$, on a $\mathcal{W}_{t} \cap \operatorname{Vect}(U)=\left\{u_{1},-u_{1}\right\}$ et $\sigma(U)=y 2^{t}+y 2^{t+1-\left|u_{1}\right|}+t-1$. Cette quantité n'excède pas $2^{2 t-1} y-1$ lorsque $t \geqslant 3$; elle est $\leqslant 2^{2 t-1} y$ lorsque $t=2$. Dans ce dernier cas, les relations $\left|u_{1}\right|=2$ et $2^{t-1} y>1$ impliquent

$$
J_{y}(X ; U) \ll X^{\sigma(U)} \int_{\mathbb{R}^{t}}\left(\frac{1}{1+\left|u_{1}(\boldsymbol{\vartheta})\right|}\right)^{2^{t-1} y} \frac{\mathrm{d} \vartheta_{1} \cdots \mathrm{d} \vartheta_{t}}{\prod_{j<h \leqslant t}\left(1+\vartheta_{h}^{\delta}\right)} \ll X^{\sigma(U)},
$$

d'où la majoration requise dans cette circonstance. 
Soit alors $j \geqslant 2$. Pour tous $g \in[1, t], h \in] j, t], g \neq h$, l'une au plus des deux formes $e_{g}^{*}+e_{h}^{*}$, $e_{g}^{*}-e_{h}^{*}$ appartient à $\operatorname{Vect}(U)$. Ainsi

$$
\sum_{\substack{w \in \mathcal{W}_{t}, w \notin \operatorname{Vect}(U) \\|w|=2}} 1 \geqslant 2 j(t-j)+(t-j)(t-j-1)=(t-j)(t+j-1),
$$

d'où

$$
\sigma(U)+j \leqslant y 2^{2 t-1}-y(t-j)(t+j-1) 2^{t-2}+t \leqslant 2^{2 t-1} y,
$$

lorsque $y>2^{1-t}, t \geqslant 2,2 \leqslant j \leqslant t-1$ sauf si $j=t-1$ et donc $t \geqslant 3$. Dans ce dernier cas, les formes $e_{1}^{*}-e_{2}^{*}, e_{1}^{*}+e_{2}^{*}, e_{h}^{*} \pm e_{t}^{*}$ avec $h \in\{1,2\}$ non encore comptabilisées dans le minorant $(2 \cdot 18)$ ne peuvent appartenir toutes à $\operatorname{Vect}(U)$. Il s'ensuit que

$$
\sum_{\substack{w \in \mathcal{W}_{t}, w \notin \operatorname{Vect}(U) \\|w|=2}} 1 \geqslant(t-j)(t+j-1)+1
$$

et donc

$$
\sigma(U)+t-1 \leqslant y 2^{2 t-1}-y 2^{t-2}((t-j)(t+j-1)+1)+t \leqslant y 2^{2 t-1} .
$$

Lemme 2.7. Soient $s \in \mathbb{R}, t \geqslant 1, \chi$ un caractère de Dirichlet réel non principal, $A>0$, $c>0, \eta \in] 0,1\left[, y>0, z \in \mathbb{R}, y+z>0\right.$, et $g \in \mathcal{M}_{A}(\chi ; c, \eta)$ telle que $y(g)=y, z(g)=z$.

(i) Si $s>0$, nous avons, uniformément pour $k \geqslant 1$,

$$
\sum_{\substack{n \geqslant 1 \\ \omega(n)=k}} \frac{\mu(n)^{2} g(n) \tau^{*}(n, \chi)^{t}}{n\left\{\log P^{+}(n)\right\}^{s}} \ll k 2^{(2 t-1) k}\left\{\left(\frac{y}{s}\right)^{k}+\left(\frac{y+z}{s+1}\right)^{k}\right\} .
$$

(ii) Si $s>-y$, nous avons, uniformément pour $k \geqslant 1,0<\sigma \leqslant 1$,

$$
\sum_{\substack{n \geqslant 1 \\ \omega(n) \geqslant k}} \frac{\mu(n)^{2} g(n) \tau^{*}\left(n_{k}, \chi\right)^{t}}{n^{1+\sigma}\left\{\log P^{+}\left(n_{k}\right)\right\}^{s}} \ll \frac{k 2^{(2 t-1) k}}{\sigma^{y}}\left\{\left(\frac{y}{s+y}\right)^{k}+\left(\frac{y+z}{s+y+1}\right)^{k}\right\} .
$$

(iii) Nous avons, sous les mêmes hypothèses,

$$
\sum_{n \geqslant 1} \frac{\mu(n)^{2} g(n) \tau^{*}(n, \chi)^{t}}{n^{1+\sigma}} \ll \sigma^{-2^{2 t-1} y}\left\{1+\sigma^{1-z 2^{2 t-1}}\right\} \log (1 / \sigma) \quad\left(0<\sigma \leqslant \frac{1}{2}\right) .
$$

De plus, lorsque $t$ est entier, $t \geqslant 2, y>2^{1-t}$ et $z \leqslant 0$, nous pouvons remplacer le membre de droite de (2.21) par $\sigma^{t-2^{2 t-1} y}$.

Démonstration. En employant l'inégalité de Hölder lorsque $t>1$, nous pouvons écrire

$$
\tau^{*}(n, \chi)^{t} \leqslant \frac{2}{(2 \pi)^{t}} \int_{0}^{\infty} \frac{|\tau(n, \chi ; \vartheta)|^{2 t}}{6+\vartheta^{2}} \mathrm{~d} \vartheta\left(\int_{0}^{\infty} \frac{\mathrm{d} \vartheta}{6+\vartheta^{2}}\right)^{t-1} .
$$

Cette majoration est trivialement valable si $t=1$. Désignons par $T$ le membre de gauche de $(2 \cdot 19)$. Nous avons donc

$$
T \ll \int_{0}^{\infty} \frac{T(\vartheta)}{1+\vartheta^{2}} \mathrm{~d} \vartheta
$$

avec

$$
\begin{aligned}
T(\vartheta) & =\sum_{\substack{n \geqslant 1 \\
\omega(n)=k}} \frac{\mu(n)^{2} g(n)|\tau(n, \chi ; \vartheta)|^{2 t}}{n\left\{\log P^{+}(n)\right\}^{s}} \\
& \leqslant \frac{1}{(k-1) !} \sum_{p \in \mathcal{P}} \frac{g(p)|\tau(p, \chi ; \vartheta)|^{2 t}}{p(\log p)^{s}}\left(\sum_{\substack{r<p \\
r \in \mathcal{P}}} \frac{g(r)|\tau(r, \chi ; \vartheta)|^{2 t}}{r}\right)^{k-1} .
\end{aligned}
$$


Appliquons $(2 \cdot 13)$ et $(2 \cdot 14)$ en majorant $\lambda(t)$ par $2^{2 t-1}$. Il s'ensuit que, lorsque $0<\vartheta \leqslant 1$,

$$
T(\vartheta) \ll \frac{4^{t} 2^{(2 t-1) k}}{(k-1) !}\left\{T_{1}(\vartheta)+T_{2}(\vartheta)\right\},
$$

avec

$$
\begin{aligned}
& T_{1}(\vartheta):=\sum_{p \leqslant \exp (1 / \vartheta)} \frac{g(p)(y+z)^{k}\left(\log _{2} p+c_{0}\right)^{k-1}}{p(\log p)^{s}}, \\
& T_{2}(\vartheta):=\sum_{p>\exp (1 / \vartheta)} \frac{g(p)\left(y \log _{2} p+z \log (1 / \vartheta)+c_{0}\right)^{k-1}}{p(\log p)^{s}}
\end{aligned}
$$

où $c_{0}$ est une constante, choisie par exemple $\geqslant 1$, et dépendant au plus de $c, \eta, s, \chi, y, z$. Il suit

$$
\begin{aligned}
\int_{0}^{1} T_{1}(\vartheta) \mathrm{d} \vartheta & \ll(y+z)^{k} \sum_{p \in \mathcal{P}} \frac{g(p)\left(\log _{2} p+c_{0}\right)^{k-1}}{p(\log p)^{s+1}} \ll \frac{(k-1) !(y+z)^{k}}{(s+1)^{k}} \\
\int_{0}^{1} T_{2}(\vartheta) \mathrm{d} \vartheta & \ll \int_{0}^{1} \mathrm{~d} \vartheta \int_{\log (1 / \vartheta)}^{\infty}\left(y u+z \log (1 / \vartheta)+c_{0}\right)^{k-1} \mathrm{e}^{-s u} \mathrm{~d} u \\
& \ll \int_{0}^{\infty}\left(v+c_{0}\right)^{k-1} \mathrm{e}^{-s v / y} \mathrm{~d} v \int_{\exp \{-v /(y+z)\}}^{1} \vartheta^{-s z / y} \mathrm{~d} \vartheta \\
& \ll \int_{0}^{\infty}\left(v+c_{0}\right)^{k}\left\{\mathrm{e}^{-s v / y}+\mathrm{e}^{-v(s+1) /(y+z)}\right\} \mathrm{d} v \ll \frac{k ! y^{k}}{s^{k}}+\frac{k !(y+z)^{k}}{(s+1)^{k}}
\end{aligned}
$$

où les sommes en $p$ ont été évaluées par intégration par parties grâce à l'hypothèse (1·3).

Semblablement, lorsque $\vartheta>1$, nous obtenons, en posant $b:=B(t) /\{y \lambda(t)\}$,

$$
T(\vartheta) \ll \frac{y^{k} \lambda(t)^{k}}{(k-1) !} \sum_{p \in \mathcal{P}} \frac{g(p)\left\{\log _{2} p+b \log _{2}(2+\vartheta)\right\}^{k-1}}{p(\log p)^{s}} \ll(y \lambda(t) / s)^{k}\{\log (2+\vartheta)\}^{s b}
$$

La majoration $(2 \cdot 19)$ est obtenue par report de $(2 \cdot 24)$ et $(2 \cdot 27)$ dans $(2 \cdot 23)$ en tenant compte de $(2 \cdot 25)$ et $(2 \cdot 26)$.

On obtient $(2 \cdot 20)$ en notant que

$$
\sum_{n \geqslant 1} \frac{\mu(n)^{2} g(n) \tau^{*}\left(n_{k}, \chi\right)^{t}}{n^{1+\sigma}\left\{\log P^{+}\left(n_{k}\right)\right\}^{s}} \leqslant \sum_{\substack{m \geqslant 1 \\ \omega(m)=k}} \frac{\mu(m)^{2} g(m) \tau^{*}(m, \chi)^{t}}{m^{1+\sigma}\left\{\log P^{+}(m)\right\}^{s}} \prod_{p>P^{+}(m)}\left(1+\frac{g(p)}{p^{1+\sigma}}\right)
$$

et en remarquant que la relation $(2 \cdot 15)$ permet de majorer le dernier produit par

$$
\ll \sigma^{-y} P^{+}(m)^{\sigma} /\left\{\log P^{+}(m)\right\}^{y} .
$$

Nous omettons les détails de cette estimation, semblables à ceux de la démonstration du lemme 71.2 de [9].

Pour établir $(2 \cdot 21)$, nous déduisons du Lemme 2.5 que

$$
\sum_{n \geqslant 1} \frac{\mu(n)^{2} g(n)|\tau(n, \chi ; \vartheta)|^{2 t}}{n^{1+\sigma}} \ll \sigma^{-y 2^{2 t-1}}\left\{1+(\sigma+|\vartheta|)^{-z 2^{2 t-1}}\right\}\{\log (3+|\vartheta|)\}^{O(1)}
$$

et nous appliquons $(2 \cdot 22)$. 
Lorsque $t$ est entier, $t \geqslant 2, y>2^{1-t}$ et $z \leqslant 0$, nous pouvons écrire

$$
\begin{aligned}
S(\sigma) & :=\sum_{n \geqslant 1} \frac{\mu(n)^{2} g(n) \tau^{*}(n, \chi)^{t}}{n^{1+\sigma}} \\
& \ll \int_{\mathbb{R}^{t}} \sum_{n \geqslant 1} \frac{\mu(n)^{2} g(n)}{n^{1+\sigma}} \prod_{1 \leqslant j \leqslant t}\left|\tau\left(n, \chi ; \vartheta_{j}\right)\right|^{2} \prod_{1 \leqslant j \leqslant t} \frac{\mathrm{d} \vartheta_{j}}{1+\vartheta_{j}^{2}} \\
& \ll \int_{\mathbb{R}^{t}} \exp \left\{\sum_{p \in \mathcal{P}} \frac{g(p)}{p^{1+\sigma}} \prod_{1 \leqslant j \leqslant t}\left|\tau\left(p, \chi ; \vartheta_{j}\right)\right|^{2}\right\} \prod_{1 \leqslant j \leqslant t} \frac{\mathrm{d} \vartheta_{j}}{1+\vartheta_{j}^{2}} \\
& \ll \int_{\mathbb{R}^{t}} \exp \left\{\sum_{p \leqslant \exp (1 / \sigma)} \frac{g(p)}{p^{1+\sigma}} \prod_{1 \leqslant j \leqslant t}\left|\tau\left(p, \chi ; \vartheta_{j}\right)\right|^{2}\right\} \prod_{1 \leqslant j \leqslant t} \frac{\mathrm{d} \vartheta_{j}}{1+\vartheta_{j}^{2}} .
\end{aligned}
$$

À ce stade, observons que, avec les notations introduites avant l'énoncé du Lemme 2.6, nous avons, pour tout nombre premier $p$,

$$
\begin{aligned}
\prod_{1 \leqslant j \leqslant t}\left|\tau\left(p, \chi ; \vartheta_{j}\right)\right|^{2} & =\prod_{1 \leqslant j \leqslant t}\left(2+\chi(p) p^{i \vartheta_{j}}+\chi(p) p^{-i \vartheta_{j}}\right) \\
& =\sum_{w \in \mathcal{W}_{t}} 2^{t-|w|} \chi(p)^{|w|} \cos (w(\boldsymbol{\vartheta}) \log p) .
\end{aligned}
$$

En vertu de $(2 \cdot 13),(2 \cdot 14)$ et $(1 \cdot 3)$, il s'ensuit que

$$
\begin{aligned}
\sum_{p<\exp (1 / \sigma)} \frac{g(p)}{p} & \prod_{1 \leqslant j \leqslant t}\left|\tau\left(p, \chi ; \vartheta_{j}\right)\right|^{2}=\sum_{w \in \mathcal{W}_{t}} 2^{t-|w|} \sum_{p \leqslant \exp (1 / \sigma)} g(p) \chi(p)^{|w|} \frac{\cos (w(\boldsymbol{\vartheta}) \log p)}{p} \\
& \leqslant y \sum_{\substack{w \in \mathcal{W}_{t} \\
|w| \equiv 0(\bmod 2)}} 2^{t-|w|} \log \left(1+\frac{1}{\sigma+|w(\boldsymbol{\vartheta})|}\right)+O\left(\sum_{1 \leqslant j \leqslant t} \log _{2}\left(3+\left|\vartheta_{j}\right|\right)\right) .
\end{aligned}
$$

Reportons dans $(2 \cdot 28)$. Il suit

$$
S(\sigma) \ll \int_{\mathbb{R}^{t}} \prod_{\substack{w \in \mathcal{W}_{t} \\|w| \equiv 0(\bmod 2)}}\left(1+\frac{1}{\sigma+|w(\boldsymbol{\vartheta})|}\right)^{2^{t-|w|} y} \prod_{1 \leqslant j \leqslant t} \frac{\left\{\log \left(3+\left|\vartheta_{j}\right|\right)\right\}^{B} \mathrm{~d} \vartheta_{j}}{1+\vartheta_{j}^{2}}
$$

où $B:=B(t ; c, \eta)$. En opérant le changement de variables $\varphi_{j}=\vartheta_{j} / \sigma$, nous obtenons

$$
S(\sigma) \ll \sigma^{t} I_{y}\left(\frac{3}{2}, 1 / \sigma\right) .
$$

La majoration souhaitée résulte donc du Lemme 2.6.

\section{Démonstration du Théorème 1.1}

\subsection{Le cas $y \leqslant y_{0}(t):$ méthode itérative}

Le résultat suivant permet d'opérer une première réduction du problème.

Lemme 3.1. Soient $A>0, c>0, \eta \in] 0,1\left[\right.$ et $g \in \mathcal{M}_{A}(c, \eta)$. On a

$$
\mathfrak{S}_{t}(x, \chi ; g) \ll \frac{x}{\log x} \sum_{n \leqslant x} \frac{\mu(n)^{2} g(n) \Delta(n, \chi)^{2 t}}{n} \quad(x \geqslant 2) .
$$

Démonstration. Compte tenu des hypothèses $(1 \cdot 1)$ et $(1 \cdot 2)$, la preuve est pratiquement identique à celle de la formule (25) de [18]. Nous laissons les détails au lecteur, en mentionnant simplement la majoration

$$
\Delta(m n, \chi) \leqslant \tau(m) \Delta(n, \chi) \quad((m, n)=1) .
$$


Le point de départ de la démonstration est l'identité suivante, valable pour tous $n \geqslant 1$, $p \in \mathcal{P}$, tels que $p \nmid n$ :

$$
\Delta(n p, \chi ; u, v)=\Delta(n, \chi ; u, v)+\chi(p) \Delta(n, \chi ; u-\log p, v) .
$$

Par élévation à la puissance $2 q$ et développement binomial, il suit, avec la notation $(2 \cdot 11)$,

$$
M_{2 q}(n p, \chi)=2 M_{2 q}(n, \chi)+\sum_{1 \leqslant j \leqslant 2 q-1}\left(\begin{array}{c}
2 q \\
j
\end{array}\right) \chi(p)^{2 q-j} N_{j, 2 q}(n, \log p) .
$$

En notant que

$$
\begin{aligned}
N_{2 h+1,2 q}(n, w) & \leqslant \frac{1}{2} N_{2 h+2,2 q}(n, w)+\frac{1}{2} N_{2 h, 2 q}(n, w) \quad(1 \leqslant h \leqslant q-2), \\
N_{1,2 q}(n, w) & \leqslant \frac{1}{2} q N_{2,2 q}(n, w)+N_{0,2 q}(n, w) /(2 q), \\
N_{2 q-1,2 q}(n, w) & \leqslant N_{2 q, 2 q}(n, w) /(2 q)+\frac{1}{2} q N_{2 q-2,2 q}(n, w),
\end{aligned}
$$

nous obtenons

$$
M_{2 q}(n p, \chi) \leqslant 4 M_{2 q}(n, \chi)+W_{2 q}(n, p)
$$

avec

$$
W_{2 q}(n, p):=(1+q) \sum_{1 \leqslant j \leqslant q-1}\left(\begin{array}{l}
2 q \\
2 j
\end{array}\right) N_{2 j, 2 q}(n, \log p) .
$$

Posons

$$
L_{k, q}(\sigma):=\sum_{n \geqslant 1} \frac{\mu(n)^{2} g(n) M_{2 q}\left(n_{k}, \chi\right)^{t / q}}{n^{1+\sigma}} .
$$

Grâce à l'inégalité de Minkowski, nous déduisons de (3·2) appliquée avec $n=n_{k}, p=p_{k+1}=$ $p_{k+1}(n)$, que, si $\omega(n)>k, q \geqslant t$, nous avons

$$
L_{k+1, q}(\sigma) \leqslant 4^{t / q} L_{k, q}(\sigma)+\sum_{\substack{m \geqslant 1 \\ \omega(m)=k}} \sum_{p>P^{+}(m)} W_{2 q}(m, p)^{t / q} \sum_{n_{k+1}=m p} \frac{\mu(n)^{2} g(n)}{n^{1+\sigma}} .
$$

La somme intérieure n'excède pas

$$
\frac{\mu(m p)^{2} g(m p)}{(m p)^{1+\sigma}} \mathcal{G}(\sigma) \prod_{\substack{r \leqslant p \\ r \in \mathcal{P}}}\left(1+\frac{g(r)}{r^{1+\sigma}}\right)^{-1} \ll \frac{\mu(m p)^{2} g(m) g(p)}{m^{1+\sigma} p^{1+\sigma}\left(1-p^{-\sigma}\right)^{y}} \ll \frac{\mu(m p)^{2} g(m) g(p)}{m^{1+\sigma} p(\sigma \log p)^{y}},
$$

où nous avons utilisé l'évaluation

$$
\sum_{\substack{r \leqslant p \\ p \in \mathcal{P}}} \frac{g(r)}{r^{1+\sigma}}=y \log \left(\frac{1-p^{-\sigma}}{\sigma}\right)+O(1)
$$

qui découle de (1.3) et du lemme 3.6 de [4]. Il vient donc

$$
L_{k+1, q}(\sigma)-4^{t / q} L_{k, q}(\sigma) \ll \frac{1}{\sigma^{y}} \sum_{\substack{m \geqslant 1 \\ \omega(m)=k}} \frac{\mu(m)^{2} g(m)}{m^{1+\sigma}} \sum_{p>P^{+}(m)} \frac{g(p) W_{2 q}(m, p)^{t / q}}{p(\log p)^{y}} .
$$


D'après le Lemme 2.4, nous avons

$$
\begin{aligned}
& \sum_{p>z} \frac{W_{2 q}(m, p) g(p) \log p}{p} \\
& \quad \leqslant 2 q y 4^{q}\left\{M_{2 q}(m, \chi)^{(q-2) /(q-1)} \tau^{*}(m, \chi)^{q /(q-1)}+R_{q}(m, z)\right\}
\end{aligned}
$$

et, d'après $(1 \cdot 3)$,

$$
\sum_{p>z} \frac{g(p)}{p(\log p)^{s}} \ll_{s} \frac{1}{(\log z)^{s}} \quad(s>0) .
$$

En employant ces inégalités avec $z:=P^{+}(m)$, nous obtenons que, pour $q>t$, la somme intérieure de $(3 \cdot 4)$ est

$$
\begin{aligned}
& \leqslant \sum_{p>P^{+}(m)} \frac{g(p)\left\{W_{2 q}(m, p) \log p\right\}^{t / q}}{p(\log p)^{y+t / q}} \\
& \leqslant\left\{\sum_{p>P^{+}(m)} \frac{g(p) W_{2 q}(m, p) \log p}{p}\right\}^{t / q}\left\{\sum_{p>P^{+}(m)} \frac{g(p)}{p(\log p)^{(q y+t) /(q-t)}}\right\}^{(q-t) / q} \\
& \ll \frac{M_{2 q}(m, \chi)^{t(q-2) / q(q-1)} \tau^{*}(m, \chi)^{t /(q-1)}}{\left\{\log P^{+}(m)\right\}^{y+t / q}}+\frac{M_{2 q}(m)^{t(2 q-2) / q(2 q-1)} \tau(m)^{2 t /(2 q-1)}}{\mathrm{e}^{c\left\{\log P^{+}(m)\right\}^{\eta}}} .
\end{aligned}
$$

En majorant trivialement $M_{2 q}(m)$ par $\tau(m)^{2 q}$, nous obtenons que la contribution du terme de droite à la majoration de (3.4) est

$$
\ll \frac{1}{\sigma^{y}} \sum_{\substack{m \geqslant 1 \\ \omega(m)=k}} \frac{\mu(m)^{2} g(m) \tau(m)^{2 t}}{m^{1+\sigma} \mathrm{e}^{c\left\{\log P^{+}(m)\right\}^{\eta}}} \ll \frac{1}{(k-1) ! \sigma^{y}} \sum_{p} \frac{\left(4^{t} y \log _{2} p+a\right)^{k-1}}{p \mathrm{e}^{c\{\log p\}^{\eta}}} \ll \frac{1}{\sqrt{k !} \sigma^{y}},
$$

où $a$ est une constante dépendant au plus de $t$.

Pour traiter la contribution du terme de gauche, nous remarquons que

$$
\sum_{\substack{n \geqslant 1 \\ n_{k}=m}} \frac{\mu(n)^{2} g(n)}{n^{1+\sigma}} \gg \frac{\mu(m)^{2} g(m)}{m^{1+\sigma}\left\{1-P^{+}(m)^{-\sigma}\right\}^{y}} \gg \frac{\mu(m)^{2} g(m)}{m^{1+\sigma} \sigma^{y}\left\{\log P^{+}(m)\right\}^{y}},
$$

d'où

$$
\begin{gathered}
\sum_{\substack{m \geqslant 1 \\
\omega(m)=k}} \frac{\mu(m)^{2} g(m) M_{2 q}(m, \chi)^{t(q-2) / q(q-1)} \tau^{*}(m, \chi)^{t /(q-1)}}{\sigma^{y} m^{1+\sigma}\left\{\log P^{+}(m)\right\}^{y+t / q}} \\
\ll \sum_{\substack{n \geqslant 1 \\
\omega(n) \geqslant k}} \frac{\mu(n)^{2} g(n) M_{2 q}\left(n_{k}, \chi\right)^{t(q-2) / q(q-1)} \tau^{*}\left(n_{k}, \chi\right)^{t /(q-1)}}{n^{1+\sigma}\left\{\log P^{+}\left(n_{k}\right)\right\}^{t / q}} .
\end{gathered}
$$

Employant à nouveau l'inégalité de Hölder avec exposant $(q-1) /(q-2), q-1$, il suit, lorsque $q>2$,

$$
\begin{aligned}
& \sum_{\substack{m \geqslant 1 \\
\omega(m)=k}} \frac{\mu(m)^{2} g(m) M_{2 q}(m, \chi)^{t(q-2) / q(q-1)} \tau^{*}(m, \chi)^{t /(q-1)}}{\sigma^{y} m^{1+\sigma}\left\{\log P^{+}(m)\right\}^{y+t / q}} \\
& \ll L_{k, q}(\sigma)^{(q-2) /(q-1)}\left\{\sum_{\substack{n \geqslant 1 \\
\omega(n) \geqslant k}} \frac{\mu(n)^{2} g(n) \tau^{*}\left(n_{k}, \chi\right)^{t}}{n^{1+\sigma}\left\{\log P^{+}\left(n_{k}\right)\right\}^{t(q-1) / q}}\right\}^{1 /(q-1)} \\
& \ll \frac{L_{k, q}(\sigma)^{(q-2) /(q-1)} w_{q}^{k /(q-1)}}{\sigma^{y /(q-1)}},
\end{aligned}
$$


où l'on a posé

$$
w_{q}:=2^{2 t-1} \max \{y /(y+t-t / q),(y+z) /(y+1+t-t / q)\} \leqslant 1 /(1-1 / 2 q)
$$

et où la dernière estimation découle du Lemme 2.7. Il est immédiat que cette majoration est encore valable si $q=2$. En reportant dans $(3 \cdot 4)$, nous obtenons donc

$$
L_{k+1, q}(\sigma)-4^{t / q} L_{k, q}(\sigma) \ll \frac{L_{k, q}(\sigma)^{(q-2) /(q-1)} w_{q}^{k /(q-1)}}{\sigma^{y /(q-1)}}+\frac{1}{\sqrt{k !} \sigma^{y}} .
$$

Introduisons alors

$$
L_{k, q}^{*}(\sigma):=L_{k, q}(\sigma)+4^{k t / q} \sum_{n \geqslant 1} \frac{\mu(n)^{2} g(n)}{n^{1+\sigma}}=L_{k, q}(\sigma)+4^{k t / q} \mathcal{G}(\sigma),
$$

de sorte que (3.6) est encore valable en remplaçant $L$ par $L^{*}$. Comme, en vertu de (2.6), nous disposons de la minoration

$$
L_{k, q}^{*}(\sigma) \gg 4^{k t / q} / \sigma^{y}
$$

nous pouvons écrire

$$
L_{k+1, q}^{*}(\sigma) \leqslant L_{k, q}^{*}(\sigma)\left\{4^{t / q}+B H_{q}^{-k /(q-1)}+\frac{C}{\sqrt{k !}}\right\}
$$

où l'on a posé $\left.\left.H_{q}:=(1-1 / 2 q) 4^{t / q} \in\right] 1,4^{t / q} / w_{q}\right]$ et $C$ désigne une constante convenable. Il s'ensuit que, pour $q \leqslant \sqrt{k /(1+\log k)}$,

$$
L_{k+1, q}^{*}(\sigma) \leqslant L_{k, q}^{*}(\sigma) \mathrm{e}^{c_{1} / q},
$$

où $c_{1}$ est une constante adéquate, dépendant éventuellement de $t$.

La mise en ouvre de la récurrence nécessite également une estimation de $L_{k, q+1}^{*}(\sigma)$ en fonction de $L_{k, q}^{*}(\sigma)$. À cette fin, nous employons les Lemmes 2.1 et 2.2. En effet, conservant la notation $\mathcal{A}_{k}$ introduite au Lemme 2.2 et notant $K$ une constante absolue convenable, nous avons d'après $(2 \cdot 5)$ et $(2 \cdot 7)$,

$$
\begin{aligned}
& L_{k, q+1}^{*}(\sigma) \leqslant \sum_{n \in \mathcal{A}_{k}} \frac{\mu(n)^{2} g(n) M_{2 q+2}\left(n_{k}, \chi\right)^{t /(q+1)}}{n^{1+\sigma}} \\
& \quad+\sum_{n \in \mathbb{N}^{*} \backslash \mathcal{A}_{k}} \frac{\mu(n)^{2} g(n) \tau\left(n_{k}\right)^{2 t}}{n^{1+\sigma}}+4^{k t / q} \mathcal{G}(\sigma) \\
& \leqslant 2^{8 t /(q+1)} 420^{2 k t^{2} / y q(q+1)} \sum_{n \in \mathcal{A}_{k}} \frac{\mu(n)^{2} M_{2 q}\left(n_{k}, \chi\right)^{t / q}}{n^{1+\sigma}}+\left(K \mathrm{e}^{-k t / 3}+4^{t k / q}\right) \mathcal{G}(\sigma) \\
& \leqslant\left(1+K \mathrm{e}^{-k t / 3}\right) 2^{8 t /(q+1)} 420^{2 k t^{2} / y q(q+1)} L_{k, q}^{*}(\sigma)
\end{aligned}
$$

où nous avons utilisé l'inégalité $\frac{16}{9}-\log 4>\frac{1}{3}$. Dans ce qui suit, nous désignons par $c_{j}(j \geqslant 2)$ des constantes positives, dépendant éventuellement de $\chi, t$ et $y$. Posons

$$
q(k):=\lfloor\sqrt{k /(1+\log k)}\rfloor .
$$

Soit $k_{0}:=\lfloor t\rfloor+1$. Nous cherchons à estimer $L_{k, q(k)}(\sigma)$ en fonction de $L_{k_{0}, q\left(k_{0}\right)}(\sigma) \ll 1 / \sigma^{y}$. À cette fin, nous employons tour à tour $(3 \cdot 7)$, pour faire décroître la valeur de $k$, et $(3 \cdot 8)$ 
pour assurer la relation de compatibilité $q \leqslant \sqrt{k / \log k}$. La première manipulation fournit un facteur

$$
\leqslant \prod_{1 \leqslant q \leqslant q(k)} q^{c_{1}} \leqslant \mathrm{e}^{c_{2} \sqrt{k \log k}}
$$

alors que la seconde manipulation induit un facteur global n'excédant pas

$$
\prod_{1 \leqslant q \leqslant q(k)}\left\{\left(1+K \mathrm{e}^{-q^{2} t / 3}\right) 2^{8 t /(q+1)} \mathrm{e}^{c_{3} \log k}\right\} \leqslant \mathrm{e}^{c_{4} \sqrt{k \log k}} .
$$

Ainsi

$$
L_{k, q(k)}^{*}(\sigma) \ll \frac{\mathrm{e}^{c_{5} \sqrt{k \log k}}}{\sigma^{y}} .
$$

En choisissant, par exemple, $k=M:=\left\lfloor 4^{t+1} y \log _{2} x\right\rfloor$ et $\sigma:=1 / \log x$, nous obtenons donc

$$
L_{M, q(M)}(\sigma) \ll_{\varepsilon}(\log x)^{y} \mathrm{e}^{c_{6} \sqrt{\log _{2} x \log _{3} x}} .
$$

Observons que

$$
E^{*}\left(n_{M}\right)^{-2 / q(M)} \ll \mathrm{e}^{c_{7} \sqrt{\log _{2} x \log _{3} x}}
$$

lorsque $n \in \mathcal{A}_{M}$ et que, d'après $(2 \cdot 7)$,

$$
\sum_{n \in \mathbb{N}^{*} \backslash \mathcal{A}_{M}} \frac{g(n) \tau\left(n_{M}\right)^{2 t}}{n^{1+\sigma}} \ll 1 .
$$

D'après le Lemme 2.1, la majoration (3·10) est donc en fait également valable, à la valeur de $c_{6}$ près, pour $\sum_{n \leqslant x} \mu(n)^{2} g(n) \Delta\left(n_{M}, \chi\right)^{2 t} / n$, et donc aussi pour

$$
\sum_{\substack{n \leqslant x \\ \omega(n) \leqslant M}} \frac{\mu(n)^{2} g(n) \Delta(n, \chi)^{2 t}}{n} .
$$

Comme

$$
\sum_{\substack{n \leqslant x \\ \omega(n)>M}} \frac{\mu(n)^{2} g(n) \tau(n)^{2 t}}{n} \leqslant \sum_{n \leqslant x} \frac{\mu(n)^{2} g(n) \tau(n)^{2 t} 4^{\omega(n)-M}}{n} \ll(\log x)^{4^{t+1} y(1-\log 4)},
$$

nous pouvons finalement écrire

$$
\sum_{n \leqslant x} \frac{\mu(n)^{2} g(n) \Delta(n, \chi)^{2 t}}{n} \ll(\log x)^{y} \mathrm{e}^{c_{8} \sqrt{\log _{2} x \log _{3} x}} .
$$

Le résultat annoncé en découle, grâce au Lemme 3.1.

\subsection{Le cas $y>y_{0}(t)$ : inégalités différentielles}

Le lemme suivant est une variante du lemme 70.2 de [9]; la démonstration est identique.

Lemme 3.2. Soient $L(\sigma), X(\sigma)$ des fonctions de classe $\mathcal{C}^{1}$ sur $\left.] 0, \sigma_{0}\right]$, telles que

$$
-L^{\prime}(\sigma) \leqslant \varphi(\sigma, L(\sigma)), \quad-X^{\prime}(\sigma) \geqslant \varphi(\sigma, X(\sigma)),
$$

où $\varphi(\sigma, x)$ est, pour chaque $\sigma$ fixé, une fonction croissante, au sens large, de $x$. Si $L\left(\sigma_{0}\right) \leqslant$ $X\left(\sigma_{0}\right)$, alors $L(\sigma) \leqslant X(\sigma)$ pour $0<\sigma \leqslant \sigma_{0}$.

Nous nous donnons un paramètre $T \geqslant 1$, et considérons la série

$$
L_{T, q}(\sigma):=\sum_{n \geqslant 1} \frac{\mu(n)^{2} g(n) M_{2 q}(n, \chi)^{t / q}}{a_{n} b_{n}^{1+\sigma}},
$$

où l'on a posé

$$
a_{n}:=\prod_{p \mid n, p \leqslant T} p, \quad b_{n}:=\prod_{p \mid n, p>T} p .
$$


Sous l'hypothèse $q>t$, nous avons, pour une constante absolue convenable $a$,

$$
\begin{aligned}
-L_{T, q}^{\prime}(\sigma) & =\sum_{n \geqslant 1} \frac{\mu(n)^{2} g(n) M_{2 q}(n, \chi)^{t / q} \log b_{n}}{a_{n} b_{n}^{1+\sigma}} \\
& \leqslant \sum_{n \geqslant 1} \frac{\mu(n)^{2} g(n)}{a_{n} b_{n}^{1+\sigma}} \sum_{p>T} \frac{M_{2 q}(n p, \chi)^{t / q} g(p) \log p}{p^{1+\sigma}} \\
& \leqslant 4^{t / q} \frac{L_{T, q}(\sigma)\{y+a \sigma\}}{\sigma}+\sum_{n \geqslant 1} \frac{\mu(n)^{2} g(n)}{a_{n} b_{n}^{1+\sigma}} \sum_{p>T} \frac{W_{2 q}(n, p)^{t / q} g(p) \log p}{p^{1+\sigma}}
\end{aligned}
$$

d'après $(3 \cdot 2)$ et l'estimation

$$
\sum_{p} \frac{g(p) \log p}{p^{1+\sigma}}=\frac{y}{\sigma}+O(1)
$$

qui découle de $(1 \cdot 3)$.

D'après $(3 \cdot 5)$, la dernière somme en $p$ n'excède pas

$$
\begin{aligned}
& \left\{\sum_{p>T} \frac{W_{2 q}(n, p) g(p) \log p}{p}\right\}^{t / q}\left\{\sum_{p} \frac{g(p) \log p}{p^{1+\sigma}}\right\}^{(q-t) / q} \\
& \ll \frac{M_{2 q}(n, \chi)^{t(q-2) / q(q-1)} \tau^{*}(n, \chi)^{t /(q-1)}}{\sigma^{1-t / q}}+\frac{M_{2 q}(n)^{t(2 q-2) / q(2 q-1)} \tau(n)^{2 t /(2 q-1)}}{\mathrm{e}^{c\{\log T\}^{\eta}} \sigma^{1-t / q}} .
\end{aligned}
$$

Nous estimons la contribution du premier terme en appliquant l'inégalité de Hölder avec exposants $q-1,(q-1) /(q-2)$ (lorsque $q>2)$ et en utilisant la majoration $(2 \cdot 21)$ en notant que notre hypothèse sur $z(g)$ permet d'omettre le terme impliquant le paramètre $z$. Pour estimer celle du terme de droite, nous nous contentons de majorer $M_{2 q}(n) \operatorname{par} \tau(n)^{2 q}$. Nous obtenons donc, pour des constantes convenables $C_{0}$ et $C_{1}$

$$
\begin{aligned}
-L_{T, q}^{\prime}(\sigma) \leqslant & 4^{t / q} \frac{L_{T, q}(\sigma)\{y+a \sigma\}}{\sigma}+C_{0} \frac{L_{T, q}(\sigma)^{(q-2) /(q-1)}}{\sigma^{1-t / q+2^{2 t-1} y /(q-1)}} \\
& +\frac{C_{0} \mathrm{e}^{-c\{\log T\}^{\eta}}}{\sigma^{1-t / q}} \sum_{n \geqslant 1} \frac{\mu(n)^{2} g(n) \tau(n)^{2 t}}{a_{n} b_{n}^{1+\sigma}} \\
\leqslant & 4^{t / q} \frac{L_{T, q}(\sigma)\{y+a \sigma\}}{\sigma}+C_{0} \frac{L_{T, q}(\sigma)^{(q-2) /(q-1)}}{\sigma^{1-t / q+2^{2 t-1} y /(q-1)}}+\frac{C_{1}(\log T)^{4^{t} y} \mathrm{e}^{-c\{\log T\}^{\eta}}}{\sigma^{4^{t} y+1-t / q}} .
\end{aligned}
$$

Notant

$$
\varepsilon:=C_{1}(\log T)^{4^{t} y} \mathrm{e}^{-c(\log T)^{\eta}}, \quad \beta:=4^{t} y-\frac{t}{q},
$$

nous déduisons de ce qui précède que

$$
-L_{T, q}^{\prime}(\sigma) \leqslant \varphi\left(\sigma, L_{T, q}(\sigma)\right)
$$

avec

$$
\varphi(\sigma, x):=\frac{4^{t / q} x(1+a \sigma)}{\sigma}+\frac{C_{0} x^{(q-2) /(q-1)}}{\sigma^{1-t / q+2^{2 t-1} y /(q-1)}}+\frac{\varepsilon}{\sigma^{\beta+1}} .
$$

Posons

$$
\gamma:=2^{2 t-1} y-t+\frac{t}{q}, \quad X(\sigma):=\frac{K}{\sigma^{\gamma}}+\frac{\varepsilon}{\sigma^{\beta}},
$$

où $K$ est une constante suffisamment grande dont la valeur sera précisée plus loin. Notons que

$$
1-\frac{t}{q}+\frac{2^{2 t-1} y}{q-1}+\frac{q-2}{q-1} \gamma=\gamma+1
$$


Soit $\sigma_{0}$ une constante absolue assez petite. Sous les hypothèses

$$
\begin{aligned}
& \gamma>4^{t / q}\left(y+a \sigma_{0}\right)+C_{0} K^{-1 /(q-1)}, \\
& \beta>4^{t / q}\left(y+a \sigma_{0}\right)+C_{0} K^{-1 /(q-1)}+1,
\end{aligned}
$$

nous avons alors, pour $\sigma \leqslant \sigma_{0}$,

$$
\begin{aligned}
& -X^{\prime}(\sigma)=\frac{K \gamma}{\sigma^{\gamma+1}}+\frac{\varepsilon \beta}{\sigma^{\beta+1}} \\
& \geqslant \frac{4^{t / q}(1+a \sigma) K}{\sigma^{\gamma+1}}+\frac{4^{t / q}(1+a \sigma) \varepsilon}{\sigma^{\beta+1}}+\frac{C_{0} K^{(q-2) /(q-1)}}{\sigma^{\gamma+1}}\left(1+\frac{\varepsilon \sigma^{\gamma}}{K \sigma^{\beta}}\right)+\frac{\varepsilon}{\sigma^{\beta+1}} \\
& \geqslant \frac{4^{t / q}(1+a \sigma) K}{\sigma^{\gamma+1}}+\frac{4^{t / q}(1+a \sigma) \varepsilon}{\sigma^{\beta+1}}+\frac{C_{0} K^{(q-2) /(q-1)}}{\sigma^{\gamma+1}}\left(1+\frac{\varepsilon \sigma^{\gamma}}{K \sigma^{\beta}}\right)^{(q-2) /(q-1)}+\frac{\varepsilon}{\sigma^{\beta+1}} \\
& =\varphi(\sigma, X(\sigma)) .
\end{aligned}
$$

Comme $\gamma>y, \beta>4^{t} y-1$, les conditions (3.12) sont réalisées, pour $q$ assez grand, dès que $K \geqslant C_{2}^{q}$, où $C_{2}$ dépend au plus de $a, C_{0}, y, \sigma_{0}$. Par ailleurs, la majoration triviale

$$
L_{T, q}(\sigma) \leqslant \sum_{n \geqslant 1} \frac{\mu(n)^{2} g(n) \tau(n)^{2 t}}{a_{n} b_{n}^{1+\sigma}} \ll \frac{(\log T)^{4^{t} y}}{\sigma^{4^{t} y}},
$$

implique $L_{T, q}\left(\sigma_{0}\right) \leqslant X\left(\sigma_{0}\right)$ avec $K:=C_{2}^{q}(\log T)^{4^{t} y}$, quitte à modifier la valeur de $C_{2}$. Dans ces conditions, nous sommes donc en mesure d'appliquer le Lemme 3.2, qui fournit l'estimation

$$
L_{T, q}(\sigma) \ll \frac{C_{2}^{q}(\log T)^{4^{t} y}}{\sigma^{2^{2 t-1} y-t+t / q}}+\frac{C_{1}(\log T)^{4^{t} y} \mathrm{e}^{-c(\log T)^{\eta}}}{\sigma^{4^{t} y-t / q}} \quad\left(0<\sigma \leqslant \sigma_{0}\right) .
$$

En choisissant $T:=\exp \left\{\left(\left\{2 \cdot 4^{t} y / c\right\} \log 1 / \sigma\right)^{1 / \eta}\right\}$, nous obtenons

$$
L_{q}(\sigma):=\sum_{n \geqslant 1} \frac{\mu(n)^{2} g(n) M_{2 q}(n, \chi)^{t / q}}{n^{1+\sigma}} \leqslant L_{T, q}(\sigma) \ll \frac{C_{2}^{q}(\log 1 / \sigma)^{4^{t} y / \eta}}{\sigma^{2^{2 t-1} y-t+t / q}} .
$$

Compte tenu de $(2 \cdot 4)$ et de $(2 \cdot 8)$, nous pouvons écrire

$$
\begin{aligned}
\sum_{n \geqslant 1} \frac{\mu(n)^{2} g(n) \Delta(n, \chi)^{2 t}}{n^{1+\sigma}} & \ll \sum_{n \geqslant 1} \frac{\mu(n)^{2} g(n)}{n^{1+\sigma}}+\frac{L_{q}(\sigma)}{\sigma^{6 t \cdot 4^{t} y / q}}+\sum_{\substack{n \geqslant 1 \\
E(n) \leqslant \sigma^{3 \cdot 4^{t} y}}} \frac{\mu(n)^{2} g(n) \tau(n)^{2 t}}{n^{1+\sigma}} \\
& \ll \frac{1}{\sigma^{y}}+\frac{C_{2}^{q}(\log 1 / \sigma)^{4^{t} y / \eta}}{\sigma^{2^{2 t-1} y-t+6 t \cdot 4^{t} y / q}}+\sqrt{\sigma} \ll \frac{C_{2}^{q}(\log 1 / \sigma)^{4^{t} y / \eta}}{\sigma^{2^{2 t-1} y-t+6 t \cdot 4^{t} y / q}} .
\end{aligned}
$$

Choisissant $q \asymp \sqrt{\log 1 / \sigma}$, nous obtenons

$$
\sum_{n \geqslant 1} \frac{\mu(n)^{2} g(n) \Delta(n, \chi)^{2 t}}{n^{1+\sigma}} \ll \frac{\mathrm{e}^{\alpha \sqrt{\log 1 / \sigma}}}{\sigma^{2^{t-1} y-t}},
$$

où $\alpha$ est une constante convenable.

L'estimation (1.5) découle de cette estimation en spécialisant $\sigma:=1 / \log x$ et en faisant appel au Lemme 3.1.

Lorsque $t$ est entier $\geqslant 2$ et $y>2^{1-t}$, nous pouvons utiliser le raffinement de $(2 \cdot 21)$ énoncé au Lemme 2.7(iii) pour gagner un facteur $\sigma^{t /(q-1)}$ dans la majoration de la contribution de $(3 \cdot 11)$ à $-L_{T, q}^{\prime}(\sigma)$. Les calculs peuvent alors être menés similairement pour fournir $(1 \cdot 6)$, la condition supplémentaire $y>2 y_{0}(t)$ étant alors nécessaire pour assurer l'inégalité $\gamma>y$. 


\section{Preuves des Théorèmes 1.2 et 1.5}

L'idée de base de la démonstration est semblable à celle du théorème 60 de [9] dans le cas $y=1$, ou de l'exercice 209 de [21].

Considérons d'abord le cas de $\Delta(n, \chi)$. Pour $t \geqslant 1, y>0$, désignons par $\beta(t, y)$ le plus grand exposant pour lequel nous avons

$$
S_{t}(x, y):=\mathfrak{S}_{t}\left(x, \chi ; y^{\omega}\right) \gg x(\log x)^{\beta(t, y)} .
$$

L'inégalité $\beta(t, t) \geqslant y-1$ résulte trivialement de la minoration $\Delta(n, \chi) \geqslant 1$ valable pour tout entier $n \geqslant 1$.

L'inégalité $\beta(t, y) \geqslant 2^{2 t-1} y-2 t-1$ provient, via une estimation standard de valeur moyenne de fonction arithmétique, de la minoration

$$
\Delta(n, \chi) \geqslant \frac{h(n) \tau(n)}{1+\log n} \quad(n \geqslant 1)
$$

où $h$ est la fonction indicatrice de l'ensemble des entiers dont tous les facteurs premiers $p$ vérifient $\chi(p)=1$.

Il reste à établir l'inégalité $\beta(t, y) \geqslant 2^{t} y-t-1$. À cette fin, nous déduisons de $(2 \cdot 10)$ que l'on a, pour tout entier $n \geqslant 1$,

$$
\begin{aligned}
\frac{1}{24 \pi} \int_{-1}^{1}\left(1-\frac{\vartheta^{2}}{20}\right)|\tau(n, \chi ; \vartheta)|^{2} \mathrm{~d} \vartheta & \leqslant \frac{1}{4 \pi} \int_{\mathbb{R}}\left(1-\frac{\sin \vartheta}{\vartheta}\right) \frac{|\tau(n, \chi ; \vartheta)|^{2}}{\vartheta^{2}} \mathrm{~d} \vartheta=M_{2}(n, \chi) \\
& =\int_{-1-\log n}^{1+\log n} \int_{0}^{1} \Delta(n, \chi ; u, v)^{2} \mathrm{~d} u \mathrm{~d} v \\
& \leqslant 2(1+\log n) \Delta(n, \chi)^{2} .
\end{aligned}
$$

Posons $I(n):=\int_{-1}^{1}|\tau(n, \chi ; \vartheta)|^{2} \mathrm{~d} \vartheta$, de sorte que

$$
\Delta(n, \chi)^{2} \gg I(n) / \log x \quad(1 \leqslant n \leqslant x) .
$$

Lorsque $t>1$, nous pouvons donc écrire

$$
\begin{aligned}
\sum_{n \leqslant x} \mu(n)^{2}\left(2^{t-1} y\right)^{\omega(n)} I(n) & \leqslant\left(\sum_{n \leqslant x}\left(2^{t} y\right)^{\omega(n)}\right)^{1-1 / t}\left(\sum_{n \leqslant x} \mu(n)^{2} y^{\omega(n)} I(n)^{t}\right)^{1 / t} \\
& \ll x^{(t-1) / t}(\log x)^{\left(2^{t} y-1\right)(t-1) / t} S_{t}(x, y)^{1 / t} \log x,
\end{aligned}
$$

d'où

$$
S_{t}(x, y) \gg \frac{x}{(\log x)^{2^{t}(t-1) y+1}}\left(\int_{-1}^{1} V(x ; \vartheta) \mathrm{d} \vartheta\right)^{t} \quad(x \geqslant 2),
$$

avec, notant $z:=2^{t-1} y$,

$$
V(x ; \vartheta):=\frac{1}{x} \sum_{n \leqslant x} \mu(n)^{2} z^{\omega(n)}|\tau(n, \chi ; \vartheta)|^{2} .
$$

La minoration $(4 \cdot 2)$ est clairement encore valable lorsque $t=1$.

Soit $F(s)$ la série de Dirichlet associée à cette fonction sommatoire. Nous avons le développement en produit eulérien

$$
\begin{aligned}
F(s) & :=\sum_{n \geqslant 1} \frac{\mu(n)^{2} z^{\omega(n)}|\tau(n, \chi ; \vartheta)|^{2}}{n^{s}}=\prod_{p \in \mathcal{P}}\left(1+\frac{2 z}{p^{s}}+\frac{z \chi(p)}{p^{s+i \vartheta}}+\frac{z \chi(p)}{p^{s-i \vartheta}}\right) \\
& =\zeta(s)^{2 z} L(s+i \vartheta, \chi)^{z} L(s-i \vartheta, \chi)^{z} H(s ; \vartheta),
\end{aligned}
$$


où $H(s ; \vartheta)$ est une série convergente et uniformément bornée dans le demi-plan $\sigma \geqslant \frac{3}{4}$, vérifiant en outre

$$
|H(1 ; \vartheta)| \gg 1 \quad(|\vartheta| \leqslant 1) .
$$

Lorsque $C / \log x<|\vartheta| \leqslant 1$, où $C$ est une constante assez grande, la méthode de SelbergDelange (cf., par exemple, le théorème II.5.2 de [21], et l'exercice 208 de [21] pour les détails) fournit

$$
V(x ; \vartheta) \gg(\log x)^{2 z-1}|L(1+i \vartheta, \chi)|^{2 z} \gg_{\chi}(\log x)^{2 z-1} .
$$

En reportant dans $(4 \cdot 2)$, cette minoration implique immédiatement le résultat annoncé.

La démonstration est analogue dans le cas de $\Delta(n, \mu)$. Il suffit de remplacer, dans l'analyse précédente, $\tau(n, \chi ; \vartheta)$ par $\tau(n, \mu ; \vartheta)$ et d'observer que nous avons d'une part

$$
(\log n) \Delta(n, \mu)^{2} \gg \int_{-1}^{1}|\tau(n, \mu ; \vartheta)|^{2} \mathrm{~d} \vartheta \quad(n>1),
$$

et d'autre part, pour tout $z>0$,

$$
\sum_{n \geqslant 1} \frac{\mu(n)^{2} z^{\omega(n)}|\tau(n, \mu ; \vartheta)|^{2}}{n^{s}}=\frac{\zeta(s)^{2 z} J(s ; \vartheta)}{\zeta(s+i \vartheta)^{z} \zeta(s-i \vartheta)^{z}}
$$

où $J(s ; \vartheta)$ est une série de Dirichlet convergente et uniformément bornée dans le demi-plan $\sigma \geqslant \frac{3}{4}$, vérifiant en outre $J(1 ; \vartheta) \mid \gg 1$ pour $|\vartheta| \leqslant 1$. Nous omettons les détails résiduels.

\section{Preuve du Théorème 1.3}

La preuve étant essentiellement identique à celle du Théorème 1.1, nous nous contentons d'indications sommaires.

Les Lemmes $2.1,2.2,2.3$ et 2.4 sont valables en remplaçant $\chi$ par $\mu$. Il en va de même du Lemme 2.5 en posant formellement $z:=-y$. Ainsi, nous pouvons remplacer l'estimation $(2 \cdot 13)$ par

$$
\sum_{p \leqslant x} \frac{g(p)|\tau(p, \mu ; \vartheta)|^{2 t}}{p}=y \lambda(t) \log (1+|\vartheta| \log x)+O(1) \quad(|\vartheta| \leqslant 1)
$$

où $\lambda(t)$ est toujours la quantité définie en (1.13), et (2·14) par

$$
\sum_{p \leqslant x} \frac{g(p)|\tau(p, \mu ; \vartheta)|^{2 t}}{p} \leqslant \lambda(t) \log _{2} x+B(t) \log _{2}(2+|\vartheta|) \quad(|\vartheta|>1)
$$

où $B(t)$ est une constante convenable. On obtient ainsi que $(2 \cdot 19),(2 \cdot 20)$ et $(2 \cdot 21)$ sont valables pour $\mu$ en remplaçant $2^{2 t-1}$ par $\lambda(t)$. Notons que la dernière assertion du Lemme 2.7 devient caduque dans le cas de $\Delta(n, \mu)$ puisque $\lambda(t) \leqslant 2^{2 t-1}-t$ pour $t \geqslant 2$, l'inégalité étant stricte dès que $t>2$.

Le Lemme 3.1 est alors valable avec la même démonstration et la fin de la preuve est inchangée.

\section{Bibliographie}

[1] R. de la Bretèche \& T.D. Browning, Binary linear forms as sums of two squares, Compositio Mathematicae, 144 (6), (2008), 1375-1402.

[2] R. de la Bretèche \& T.D. Browning, Binary forms of two squares and Châtelet surfaces, Israel Journal of Math., à paraître.

[3] R. de la Bretèche, T.D. Browning \& E. Peyre, On Manin's conjecture for a family of Châtelet surfaces, Annals of Math. 175 (2012), 1-47. 
[4] R. de la Bretèche \& G. Tenenbaum, Propriétés statistiques des entiers friables, Ramanujan J. 9 (2005), 139-202.

[5] R. de la Bretèche \& G. Tenenbaum, Conjecture de Manin pour certaines surfaces de Châtelet, prépublication.

[6] P. Erdős, On the density of some sequences of integers, Bull. Amer. Math. Soc. 54 (1948), 685-692.

[7] S. Daniel, On the divisor-sum problem for binary forms, J. reine angew. Math. 507 (1999), 107-129.

[8] R.R. Hall \& G. Tenenbaum, The average orders of Hooley's $\Delta_{r}$-functions, II, Compositio Math. 60 (1986), 163-186.

[9] R.R. Hall \& G. Tenenbaum, Divisors, Cambridge tracts in mathematics, $n^{\circ}$ 90, Cambridge University Press (1988).

[10] D. R. Heath-Brown, Linear relations amongst sums of two squares. Number theory and algebraic geometry, 133-176, London Math. Soc. Lecture Note Ser. 303 CUP, 2003.

[11] C. Hooley, On a new technique and its applications to the theory of numbers, Proc. London Math. Soc. (3) 38 (1979), $\mathrm{n}^{\circ} 1,115-151$.

[12] H. Maier, On the Möbius function, Transactions Amer. Math. Soc. 301, no 2 (1987), 649-664.

[13] H. Maier \& G. Tenenbaum, On the normal concentration of divisors, J. London Math. Soc. (2) 31 (1985), 393-400.

[14] H. Maier \& G. Tenenbaum, On the normal concentration of divisors, 2, Math. Proc. Camb. Phil. Soc. $147 \mathrm{n}^{\circ} 3$ (2009), 593-614.

[15] T. Mitsui, On the prime ideal theorem, J. Math. Soc. Japan 20 (1968), 233-247.

[16] E. Peyre, Hauteurs et mesures de Tamagawa sur les variétés de Fano, Duke Math. J. 79 (1995), 101-218.

[17] P. Shiu, A Brun-Titchmarsh theorem for multiplicative functions, J. reine angew. Math. 313 (1980), 161-170.

[18] G. Tenenbaum, Sur la concentration moyenne des diviseurs, Comment. Math. Helvetici 60 (1985), 411-428.

[19] G. Tenenbaum, Fonction $\Delta$ de Hooley et applications, Progress in Math. 63, Séminaire de Théorie des Nombres, Paris 1984-85 (Birkhäuser, 1986), 225-239.

[20] G. Tenenbaum, Sur une question d'Erdős et Schinzel, II, Inventiones Math. 99 (1990), 215-224.

[21] G. Tenenbaum, Introduction à la théorie analytique et probabiliste des nombres, troisième édition, coll. Échelles, Belin, 2008, 592 pp.

Régis de la Bretèche

Institut de Mathématiques de Jussieu

UMR 7586

Université Paris Diderot-Paris 7

UFR de Mathématiques, case 7012

Bâtiment Chevaleret

75205 Paris Cedex 13

France

breteche@math.jussieu.fr
Gérald Tenenbaum

Institut Élie Cartan

Université de Nancy 1

BP 239

54506 Vandouvre Cedex

France

gerald.tenenbaum@iecn.u-nancy.fr 